\title{
COMPUTATIONAL APPROACH TO SOLVABILITY OF REFINEMENT EQUATIONS
}

\author{
VICTOR D. DIDENKO AND BERND SILBERMANN
}

\begin{abstract}
The solvability and Fredholm properties of refinement equations in spaces of square-integrable functions are studied. Necessary and jointly necessary and sufficient conditions for the solvability of homogeneous and nonhomogeneous refinement equations are established. It is shown that in the space $L_{2}(\mathbb{R})$ the kernel space of any homogeneous equation with a non-trivial solution is infinite dimensional. Moreover, the solvability problem is reduced to the study of singular values of certain matrix sequences. These sequences arise from Galerkin approximations of auxiliary linear operators. The corresponding constructions use only the coefficients of refinement equations that generate multiresolution analysis, and the coefficients of the refinement equation studied. For the equations with polynomial symbols the most complete results are obtained if the corresponding operator is considered on an appropriate subspace of the space $L_{2}(\mathbb{R})$.
\end{abstract}

\section{INTRODUCTION}

Let $a$ be an essentially bounded measurable function on the unit circle $\mathbb{T}:=\{z \in$ $\mathbb{C}:|z|=1\}$. Using the usual identification

$$
\mathbb{T} \ni z=e^{i t} \rightarrow t \in \mathbb{R} \backslash 2 \pi \mathbb{Z},
$$

we have the correspondence $a(z) \sim a(t)$ between functions on $\mathbb{T}$ and $2 \pi$-periodic functions on the real line $\mathbb{R}$, and we can use the same letter to denote each function.

Let

$$
a_{k}=\frac{1}{2 \pi} \int_{0}^{2 \pi} a\left(e^{i t}\right) e^{-i k t} d t, \quad k \in \mathbb{Z}
$$

be the Fourier coefficients of the function $a$. The refinement or scaling equation associated with the function $a$ is a functional equation of the form

$$
f(t)=\sum_{k \in \mathbb{Z}} a_{k} f(2 t-k), \quad t \in \mathbb{R} .
$$

Throughout this paper the operator corresponding to the right-hand side of equation (1.1) is called the refinement operator and is denoted by $R_{a}$. In this context the function $a$ is referred to as the symbol of the operator $R_{a}$.

A non-trivial solution of equation (1.1) is called a refinable function. Refinable functions play an important role in wavelet analysis and approximation theory, and

Received by the editor November 16, 2007 and, in revised form, August 18, 2008.

2000 Mathematics Subject Classification. Primary 65T60; Secondary 42C40, 39B32.

Key words and phrases. Refinement equation, solvability, singular values, splitting.

The first author was supported in part by Universiti Brunei Darussalam Grants PNC2/2/RG/1(66) and PNC2/2/RG/1(72). 
the solvability of equation (1.1) and its generalizations in different normed spaces have been extensively studied in the literature [1, 3, 4, 6, 8, 9, 10, 20, 21, 22, 25, 29. It is worth noting that most of the above-mentioned papers deal with the refinement operators satisfying the following two assumptions:

(1) The number of non-zero coefficients in (1.1) is finite; i.e., equation (1.1) has the form

$$
f(t)=\sum_{k=0}^{N} a_{k} f(2 t-k), \quad a_{0} \neq 0, a_{N} \neq 0 .
$$

(2) The coefficients $a_{k}, k=0,1, \ldots, N$ satisfy the condition

$$
\Delta(a)=1
$$

where

$$
\Delta(a):=\frac{1}{2} a(0)=\frac{1}{2} \sum_{k=0}^{N} a_{k} .
$$

Then it is known [1, 10] that there exists a unique compactly supported distributional solution $f_{0}$ of (1.2), where

$$
f_{0}=\mathfrak{F}^{-1}\left(\prod_{j=1}^{\infty} a\left(-2^{-j} \cdot\right)\right), \quad f_{0}(0)=1,
$$

and $\mathfrak{F}^{-1}$ denotes the inverse Fourier transform.

For a Banach space $X$ and for a bounded linear operator $A$ on $X$, let im $X A$ and $\operatorname{ker}_{X} A$ denote the range and null space of the operator $A$ on $X$, respectively. The subscript $X$ is usually omitted if that does not cause confusion. Thus the assertion that the refinement equation (1.1) has a non-trivial solution in $X$ is equivalent to the relation $\operatorname{dim} \operatorname{ker}_{X}\left(I-R_{a}\right)>0$. A homogeneous equation is called solvable in a space $X$ if it has a non-trivial solution $f \in X$. Note that the solvability of refinement equation (1.2) in $L_{1}(\mathbb{R})$ was studied by Daubechies and Lagarias [10]. Theorem 1.1 below is a reformulation of their Theorems 2.1 and 2.3 in a form convenient for our considerations.

Theorem 1.1. Let $R_{a}$ be a refinement operator acting on the Banach space $L_{1}(\mathbb{R})$ and let $\Delta(a)$ be the complex number defined by (1.4). Then:

(1) If $|\Delta(a)| \leq 1$ and $\Delta(a) \neq 1$, then $\operatorname{dim} \operatorname{ker}_{L_{1}(\mathbb{R})}\left(I-R_{a}\right)=0$.

(2) If $\Delta(a)=1$, then $\operatorname{dim} \operatorname{ker}_{L_{1}(\mathbb{R})}\left(I-R_{a}\right)=1$ or $\operatorname{dim} \operatorname{ker}_{L_{1}(\mathbb{R})}\left(I-R_{a}\right)=0$.

(3) If $|\Delta(a)|>1$, and if equation (1.1) possesses a non-trivial $L_{1}$-solution having a compact support, then $\operatorname{dim} \operatorname{ker}_{L_{1}(\mathbb{R})}\left(I-R_{a}\right)=1$.

Condition (1.3) plays a decisive role for the solvability of (1.2) in the space $L_{1}(\mathbb{R})$, viz. even if $\Delta(a)>1$ and equation (1.2) has a compactly supported non-trivial solution, it implies that $\Delta(a)=2^{m}$ for a positive integer $m$, and the refinement equation obtained from the initial equation (1.2) by replacing $\left\{a_{n}\right\}$ by $\left\{2^{-m} a_{n}\right\}$ has a non-trivial solution [10.

One may note that condition (1.3) is not so important for the solvability of refinement equations in the space $L_{2}(\mathbb{R})$. Nevertheless, it often remains one of the main assumptions in publications on the solvability of these equations in both $L_{2}$ and other normed spaces. Thus, in [29] the existence of a compactly supported 
$L_{2}$-solution of (1.2) was linked to the existence of positive solutions of an auxiliary equation in a finite-dimensional space. A different approach to the solvability of equation (1.2) was used in [20, where $L_{p}$-solutions of (1.2) are described in terms of the $p$-norm joint spectral radius of two matrices associated with the coefficients of the symbol $a$. The same condition (1.3) is used while studying the solvability of refinement equations in the space of continuous functions [6, 11, and similar assumptions are often made in works on the solvability of matrix refinement equations (see, for example, [7, 19] and the references there). Interesting results concerning the solvability of the Haar refinement equation in $L_{2}(\mathbb{R})$ have been obtained [23, 24], where however the solvability of equation (1.1) is not directly connected to the distributional solution (1.5). In particular, Theorem 3 of [24] states that the kernel space of the equation

$$
f(x)=f(2 x)+f(2 x-1)
$$

is isomorphic to an $L_{2}$-space, so it is infinite dimensional. Moreover, it was mentioned that this result "can be extended to dilation equations which have a (non-zero $\left.L_{2}(\mathbb{R})\right)$ solution with analytic Fourier transform which decays like $|\omega|^{-p}$ for some $p>1 / 2$." The author also notes that "solutions of the form discussed in [10] are usually of this type and the dilation equations used to build Daubechies' family of orthonormal wavelet bases always are."

In [23, p. 44], the author also proves that the equation

$$
f(x)=k f(2 x)+k f(2 x-1), \quad k \in \mathbb{R}
$$

is solvable in $L_{2}(\mathbb{R})$ for any fixed $k \in[1 / \sqrt{2}, \sqrt{2}]$. The latter result is especially interesting because, except for $k=1$, none of these equations possesses compactly supported $L_{2}$-solutions. Such equations therefore remain outside the perspective of most works concerned with the solvability of equation (1.1).

In the present paper, we also do not use condition (1.3) and the corresponding distributional solution (1.5) while studying the solvability problem. For $L_{2}$-spaces, other conditions seems to be more relevant. Moreover, the polynomial symbols of (1.2) are replaced by more general symbols with uniformly convergent Fourier series, where the set of the functions which have uniformly convergent Fourier series is denoted by $U C_{\mathcal{F}}(\mathbb{T})$. Note that if $a \in U C_{\mathcal{F}}(\mathbb{T})$, then $a$ is continuous, and one can define a non-negative number $\widetilde{\Delta}(a)$ by

$$
\widetilde{\Delta}(a):=\lim _{n \rightarrow \infty} \max _{x \in[0,2 \pi]} \sqrt[n]{\prod_{j=0}^{n-1}\left|a\left(2^{j} x\right)\right|} .
$$

In $\$ 3$ we give the following description for the kernel space dimension of the operator $I-R_{a}$ considered on the space $L_{2}(R)$.

Theorem 1.2. Let $a \in U C_{\mathcal{F}}(\mathbb{T})$. The following are true:

(1) If a does not vanish in the interval $[0,2 \pi]$, then:

(a) If $\sqrt{2} \notin\left[\widetilde{\Delta}^{-1}\left(a^{-1}\right), \widetilde{\Delta}(a)\right]$, then $\operatorname{dim} \operatorname{ker}_{L_{2}(\mathbb{R})}\left(I-R_{a}\right)=0$.

(b) If $\sqrt{2} \in\left[\widetilde{\Delta}^{-1}\left(a^{-1}\right), \widetilde{\Delta}(a)\right]$, then $\operatorname{dim} \operatorname{ker}_{L_{2}(\mathbb{R})}\left(I-R_{a}\right)=\infty$ or $\operatorname{dim} \operatorname{ker}_{L_{2}(\mathbb{R})}\left(I-R_{a}\right)=0$. 
(2) If a vanishes in the interval $[0,2 \pi]$, then

(a) If $\widetilde{\Delta}(a)<\sqrt{2}$, then $\operatorname{dim} \operatorname{ker}_{L_{2}(\mathbb{R})}\left(I-R_{a}\right)=0$.

(b) If $\widetilde{\Delta}(a) \geq \sqrt{2}$, then $\operatorname{dim} \operatorname{ker}_{L_{2}(\mathbb{R})}\left(I-R_{a}\right)=\infty$ or $\operatorname{dim} \operatorname{ker}_{L_{2}(\mathbb{R})}\left(I-R_{a}\right)=0$.

Thus the situation for the space $L_{2}(\mathbb{R})$ is quite different from that for $L_{1}(\mathbb{R})$. First, the refinement equation does not have a unique solution. Even if it has a compactly supported solution, there are always infinitely many other linearly independent solutions. A brief analysis also shows, that for the symbols satisfying equation (1.3), one has

$$
\widetilde{\Delta}(a) \geq 2,
$$

so such symbols satisfy condition 2(b) of Theorem 1.2 but not necessarily condition 1(b). Therefore, for the refinement equation (1.1) satisfying condition (1.3), it is advantageous to assume that its symbol $a$ vanishes somewhere in $[0,2 \pi]$. Notice that in wavelet theory, the latter condition is often ensured by the requirement 11

$$
\sum_{k} a_{2 k}=\sum_{k} a_{2 k+1}=1
$$

Another consequence of Theorem 1.2 is the following corollary.

Corollary 1.3. The operator $I-R_{a}: L_{2}(\mathbb{R}) \mapsto L_{2}(\mathbb{R})$ is Fredholm if and only if it is invertible.

We study the solvability of equation (1.1) in the space $L_{2}(\mathbb{R})$ in more detail, but as was already mentioned the approach we use is not connected to the distributional solution (1.5). The problem is instead reduced to the study of singular values of a sequence of matrices $\left(\mathbf{R}_{n m}\right)_{n, m \in \mathbb{N}}$ introduced in (4.34). These sequences arise from matrix representations in wavelet bases of the Galerkin approximations of the operator $\left(I-R_{a}\right)^{*}\left(I-R_{a}\right)$. It turns out that the form of the matrices $\mathbf{R}_{n m}$ depends only on the coefficients of the scaling equation generating the corresponding wavelet and the coefficients of the equation tested. Let us also emphasize that numerical examples indicate that the singular values of the matrix sequences constructed for the same test scaling equation, but based on different wavelet Galerkin approximations, have similar convergence rates.

This approach is related to references [26, 27, where the dimensions of the null spaces of Toeplitz operators have been investigated. For the convenience of the reader, in $₫ 2$ we recall relevant results of [27] in a modified form that suits the situation considered here. Note that to approximate the corresponding operators, 27] uses projections on trigonometric polynomial spaces, whereas for the refinement equation (1.1), the construction based on wavelets seems to be more appropriate. Thus by using wavelet bases one obtains well-structured three-diagonal block matrices. Studying the singular values of these matrices, one can get information concerning the solvability of refinement equations. In particular, in 迎 the following result is established.

Theorem 1.4. Let $a \in U C_{\mathcal{F}}(\mathbb{T})$. If equation (1.1) is solvable in $L_{2}(\mathbb{R})$, then all sequences of singular values of matrices (4.34) converge to zero. Moreover, if the operator $I-R_{a}$ is normally solvable and the sequence of the smallest singular values of matrices (4.34) converges to zero, then equation (1.1) is solvable in the space $L_{2}(\mathbb{R})$. 
This approach also allows us to study the solvability of non-homogeneous refinement equations. In fact, necessary and sufficient conditions for the existence and uniqueness of $L_{2}$-solutions of non-homogeneous refinement equations are immediately obtained as a by-product.

Theorem 1.5. Let $a \in U C_{\mathcal{F}}(\mathbb{T})$. The non-homogeneous refinement equation

$$
f=R f+g
$$

is solvable for any right-hand side $g \in L_{2}(\mathbb{R})$, and the solution is unique if and only if there are $m_{0}, n_{0} \in \mathbb{N}$ such that the set $\left\{s_{n m}(1): n \geq n_{0}, m \geq m_{0}\right\}$, which comprises the smallest singular values of the matrices $\mathbf{R}_{n m}$ of (4.34), is bounded away from zero.

Note that criteria for the existence and uniqueness of distributional, continuous, or $L_{p}$ compactly supported solutions of the non-homogeneous equation (1.9) are established in [14] and [28] where, in contrast to Theorem 1.5] the right-hand side $g$ of (1.9) is assumed to be compactly supported.

Section 5 is devoted to a discussion of numerical tests for some refinement equations which are known to be solvable or not in the space $L_{2}(\mathbb{R})$. In particular, the Haar refinement equation is tested, and refinement equations with non-compactly supported $L_{2}$-solutions are considered.

In $\$ \S 67$ we discuss the existence and uniqueness of compactly supported solutions for polynomial symbols. In this case the corresponding matrices $\mathbf{R}_{n m}$ differ from the matrices arising in $\S \S 45$, if the corresponding equation is considered in the space $L_{2}$ on an interval $[0, N]$, then it can have only a one-dimensional kernel space. Moreover, the restriction of the refinement operator to the space $L_{2}([0, N])$ is normally solvable if and only if this restriction is a Fredholm operator. On the other hand, in the $L_{2}(\mathbb{R})$ case, Fredholmness is equivalent to invertibility.

We emphasize that the method presented here is quite universal. It can be applied to the study of $L_{2}$-solvability of multivariate homogeneous and non-homogeneous refinement equations, as well as systems of such equations. Of course, the resulting matrices $\mathbf{R}_{n m}$ and formulas to compute their entries may be more complicated.

\section{FREDHOLM PROPERTIES OF LINEAR OPERATORS}

Let $\mathcal{H}$ be a separable Hilbert space and let $\mathcal{L}(\mathcal{H})$ denote the $C^{*}$-algebra of all bounded linear operators which act in the space $\mathcal{H}$. By $\mathcal{K}(\mathcal{H})$ we denote the ideal of all compact operators from $\mathcal{L}(\mathcal{H})$. Consider a sequence $\left(P_{n}\right)_{n \in \mathbb{N}}$ of orthogonal projections on $\mathcal{H}$ such that $\operatorname{dimim} P_{n}<\infty$, and the strong limit $s-\lim _{n \rightarrow \infty} P_{n}=I$. Without loss of generality we can assume that $\operatorname{dim} \operatorname{im} P_{n}=n$. By $\mathcal{A}$ we denote the algebra generated by the sequences of the form $\left(P_{n} A P_{n}\right)$, where $A \in \mathcal{L}(\mathcal{H})$, and $\mathcal{N}$ is the family of all sequences $\left(C_{n}\right)$ with $C_{n}: \operatorname{im} P_{n} \rightarrow \operatorname{im} P_{n}$ and $\left\|C_{n}\right\| \rightarrow 0$ as $n \rightarrow \infty$. As usual, the operations on $\mathcal{A}$ are defined componentwise by inheritance of the corresponding operations on $\mathcal{L}(\mathcal{H})$. Moreover, if one provides $\mathcal{A}$ with the norm

$$
\left\|\left(A_{n}\right)\right\|=\sup _{n \in \mathbb{N}}\left\|A_{n}\right\|,
$$

then it becomes a $C^{*}$-algebra and the set

$$
\mathcal{I}:=\left\{\left(P_{n} K P_{n}\right)+\left(C_{n}\right): K \in \mathcal{K}(\mathcal{H}), \quad\left(C_{n}\right) \in \mathcal{N}\right\}
$$

forms a closed two-sided ideal in $\mathcal{A}$. 
Definition 2.1. A bounded sequence $\left(A_{n}\right), A_{n} \in \mathcal{L}\left(\operatorname{im} P_{n}\right)$ is called stable if there is an $n_{0} \in \mathbb{N}$ such that for all $n \geq n_{0}$ the operators $A_{n}: \operatorname{im} P_{n} \mapsto \operatorname{im} P_{n}$ are invertible and the sequence $\left(B_{n}\right)$,

$$
B_{n}:=\left\{\begin{array}{lll}
P_{n} & \text { if } & n<n_{0} \\
\left(A_{n}\right)^{-1} P_{n} & \text { if } \quad n \geq n_{0}
\end{array}\right.
$$

is bounded.

Stable sequences play an important role in our further considerations.

An operator $A \in \mathcal{L}(\mathcal{H})$ is called a $\Phi^{+}$(or, respectively, a $\Phi^{-}$) operator if it is normally solvable and if $\operatorname{dim} \operatorname{ker} A<\infty$ (or, respectively, $\operatorname{dim}(\mathcal{H} / \operatorname{im} A)<\infty$ ). Recall that an operator $A \in \mathcal{L}(\mathcal{H})$ is normally solvable if the range of this operator is a closed subspace of $\mathcal{H}$.

In the following, the set of all $\Phi^{+}\left(\Phi^{-}\right)$operators on $\mathcal{H}$ will be denoted by $\Phi^{+}(\mathcal{H})\left(\Phi^{-}(\mathcal{H})\right)$. If $A$ is a $\Phi^{+}$and $\Phi^{-}$operator simultaneously, then it is called a Fredholm or $\Phi$-operator, and the symbol $\Phi(\mathcal{H})$ refers to the set of all such operators acting in the Hilbert space $\mathcal{H}$.

Lemma 2.2. An operator $A \in \mathcal{L}(\mathcal{H})$ is a $\Phi^{+}$operator if and only if the coset $\left(P_{n} A^{*} A P_{n}\right)+\mathcal{I}$ is invertible in the factor algebra $\mathcal{A} / \mathcal{I}$.

Proof. Let $A \in \Phi^{+}(\mathcal{H})$. Then $A^{*} \in \Phi^{-}(\mathcal{H})$ and $A^{*} A \in \Phi(\mathcal{H})$, 16. Since both operators $A^{*} A$ and $P_{\operatorname{ker} A}$ are positive, it follows that $A^{*} A+P_{\operatorname{ker} A}$ is positive definite. Hence the sequence $\left(P_{n}\left(A A^{*}+P_{\text {ker } A}\right) P_{n}\right)$ is stable; cf. [15]. Thus, there is a bounded sequence $\left(B_{n}\right), B_{n} \in \mathcal{L}\left(\operatorname{im} P_{n}\right)$ and $n_{0} \in \mathbb{N}$ such that

$$
B_{n}\left(P_{n}\left(A A^{*}+P_{\text {ker } A}\right) P_{n}\right)=P_{n}, \quad n \geq n_{0} .
$$

Moreover, in the case at hand, simple $C^{*}$-algebra arguments show that the sequence $\left(B_{n}\right)$ belongs to the same $C^{*}$-algebra $\mathcal{A}$. Therefore, this sequence converges strongly to an operator $B \in \mathcal{L}(\mathcal{H})$, and relation (2.1) can be rewritten as

$$
\left.B_{n} P_{n} A A^{*} P_{n}=P_{n}-P_{n} B P_{\text {ker } A} P_{n}+P_{n}\left(B-B_{n}\right) P_{\text {ker } A}\right) P_{n}, \quad n \geq n_{0} .
$$

Note that the operator $B P_{\operatorname{ker} A}$ is compact whereas the sequence

$$
\left(\left(P_{n}\left(B-B_{n}\right) P_{\text {ker } A}\right) P_{n}\right)
$$

converges to zero uniformly since the operator $P_{\text {ker } A}$ is compact and $s-\lim _{n \rightarrow \infty}(B-$ $\left.B_{n}\right)=0$. Thus the sequence $\left(\left(-P_{n} B P_{\operatorname{ker} A} P_{n}+P_{n}\left(B-B_{n}\right) P_{\operatorname{ker} A}\right) P_{n}\right)$ belongs to the ideal $\mathcal{I}$, so the coset $\left(P_{n} A^{*} A P_{n}\right)+\mathcal{I}$ is invertible in $\mathcal{A} / \mathcal{I}$ from the left. The right invertibility of this coset in the factor algebra $\mathcal{A} / \mathcal{I}$ can be shown analogously, and the proof of the necessity part of Lemma 2.2 is completed.

Conversely, let the coset $\left(P_{n} A^{*} A P_{n}\right)+\mathcal{I}$ be invertible in $\mathcal{A} / \mathcal{I}$. Then there exists a sequence $\left(B_{n}\right)$ in $\mathcal{A}$ such that

$$
\left(P_{n} A^{*} A P_{n}\right)\left(B_{n}\right)-\left(P_{n}\right) \in \mathcal{I}
$$

and

$$
\left(B_{n}\right)\left(P_{n} A^{*} A P_{n}\right)-\left(P_{n}\right) \in \mathcal{I} .
$$

The latter two inclusions are equivalent to the existence of compact operators $K_{1}, K_{2} \in \mathcal{K}$ and two sequences $\left(C_{n}^{1}\right),\left(C_{n}^{2}\right) \in \mathcal{N}$ such that

$$
\begin{array}{ll}
P_{n} A^{*} A P_{n} B_{n}-P_{n}=P_{n} K_{1} P_{n}+C_{n}^{1}, & n \in \mathbb{N}, \\
B_{n} P_{n} A^{*} A P_{n}-P_{n}=P_{n} K_{2} P_{n}+C_{n}^{2}, & n \in \mathbb{N} .
\end{array}
$$


Letting $n$ tend to infinity in the last two expressions yields

$$
A^{*} A B=I+K_{1}
$$

and

$$
B A^{*} A=I+K_{2} .
$$

Hence $A^{*} A$ is a $\Phi$ operator, so $A^{*} \in \Phi^{-}(\mathcal{H})$ and $A \in \Phi^{+}(\mathcal{H})$, [16].

Definition 2.3. A sequence $\left(A_{n}\right) \in \mathcal{A}$ is called Fredholm if the coset $\left(A_{n}\right)+\mathcal{I}$ is invertible in $\mathcal{A} / \mathcal{I}$.

Let $M$ be an $n \times n$ matrix. By $s_{j}, j=1,2, \ldots, n$ we denote its singular values. Note that throughout this paper we always assume that singular values are ordered as $0 \leq s_{1} \leq s_{2} \leq \ldots \leq s_{n}$. Recall that the singular values of the matrix $M$ can also be found by the formula [17.

$$
s_{k}(M)=\inf _{N \in \mathcal{F}_{n-k}^{n}}\|M-N\|, \quad k=1,2, \ldots, n,
$$

where $\mathcal{F}_{n-k}^{n}$ refers to the set of all $n \times n$ matrices of rank at most $n-k$.

Definition 2.4. A sequence $\left(A_{n}\right) \in \mathcal{A}$ is said to enjoy the $k$-splitting property if there are numbers $n_{0} \in \mathbb{N}$ and $d>0$ such that for all $n \geq n_{0}$ the singular values $s_{l n}\left(A_{n}\right), l=k+1, \ldots, n$ are in the interval $[d, \infty)$ whereas for $l=1,2, \ldots, k$,

$$
\lim _{n \rightarrow \infty} s_{l n}\left(A_{n}\right)=0 .
$$

Proposition 2.5. Assume that $\left(A_{n}\right) \in \mathcal{A}$ and $s-\lim _{n \rightarrow \infty} A_{n}=A$. If the sequence $\left(A_{n}\right)$ is Fredholm, then it is subject to the $k$-splitting property and

$$
\operatorname{dim} \operatorname{ker} A=k \text {. }
$$

Proof. Let $S_{n}$ be the orthoprojection onto the subspace $P_{n} P_{\text {ker } A} P_{n}$ in im $P_{n}$. Using formula (2.2) and the relation $\left\|A_{n} S_{n}\right\| \rightarrow 0$, as $n \rightarrow \infty$ one gets

$$
\begin{aligned}
& s_{k}\left(A_{n}\right)=\inf _{B \in \mathcal{F}_{n-k}^{n}}\left\|A_{n}-B\right\| \\
& \leq \| A_{n}\left(A_{n}^{*} A_{n}\left(P_{n}-S_{n}\right)+P_{n} P_{\operatorname{ker} A} P_{n}\right)\left(A_{n}^{*} A_{n}\left(P_{n}-S_{n}\right)+P_{n} P_{\text {ker } A} P_{n}\right)^{-1} \\
& \quad-A_{n} A_{n}^{*} A_{n}\left(I-S_{n}\right)\left(A_{n}^{*} A_{n}\left(I-S_{n}\right)+P_{n} P_{\text {ker } A} P_{n}\right)^{-1} \| .
\end{aligned}
$$

Notice that $I-S_{n} \in \mathcal{F}_{n-k}^{n}$ and the sequence $\left(A_{n}^{*} A_{n}\left(I-S_{n}\right)+P_{n} P_{\text {ker } A} P_{n}\right)$ is stable. Therefore there exists a $d_{1}>0$ and an $n_{0}$ such that for all $n \geq n_{0}$ the inequality

$$
s_{k}\left(A_{n}\right) \leq d_{1}\left\|A_{n} P_{n} P_{\text {ker } A} P_{n}\right\|
$$

holds. Inequality (2.4) implies that

$$
\lim _{n \rightarrow \infty} s_{j}\left(A_{n}\right)=0
$$

for all $j=1,2, \ldots, k$.

To estimate the singular value $s_{k+1}$ we proceed as follows:

$$
\begin{aligned}
s_{1}\left(A_{n}^{*} A_{n}+P_{n} P_{\text {ker } A} P_{n}\right) & =\inf _{D \in \mathcal{F}_{n-1}^{n}}\left\|A_{n}^{*} A_{n}+P_{n} P_{\text {ker } A} P_{n}-D\right\| \\
& =\inf _{D \in \mathcal{F}_{n-1}^{n}}\left\|A_{n}^{*} A_{n}+P_{n} P_{\text {ker } A} P_{n}-D-P_{n} P_{\text {ker } A} P_{n}\right\| \\
& \leq \inf _{D \in \mathcal{F}_{n-k}^{n}}\left\|A_{n}^{*} A_{n}-D\right\|=s_{k+1}\left(A_{n}^{*} A_{n}\right) \\
& \leq\left\|A_{n}^{*}\right\| s_{k+1}\left(A_{n}\right) .
\end{aligned}
$$


Using the stability of the sequence $\left(A_{n}^{*} A_{n}+P_{n} P_{\operatorname{ker} A} P_{n}\right)$, one finds that for all sufficiently large $n$ the inequality

$$
0<d_{2} \leq s_{1}\left(A_{n}^{*} A_{n}+P_{n} P_{\text {ker } A} P_{n}\right)
$$

holds. Hence

$$
s_{k+1}\left(A_{n}\right) \geq d_{3}>0
$$

for all sufficiently large $n$ and the proof is complete.

Theorem 2.6. The following two assertions are equivalent:

(1) $A \in \Phi^{+}(\mathcal{H})$.

(2) The sequence $\left(P_{n} A^{*} A P_{n}\right)$ is subject to the $k$-splitting property with $k=$ $\operatorname{dim} \operatorname{ker} A$.

Proof. Let $A$ be a $\Phi^{+}$operator. Then Lemma 2.2 and Proposition2.5 imply that the sequence $\left(P_{n} A^{*} A P_{n}\right)$ is subject to the $k$-splitting property and $\operatorname{dim} \operatorname{ker}\left(A^{*} A\right)=k$. It remains to note that $\operatorname{dim} \operatorname{ker}\left(A^{*} A\right)=\operatorname{dim} \operatorname{ker} A$.

To prove the reverse statement, assume that $A$ is not a $\Phi^{+}$operator. Then the operator $A^{*} A$ is not Fredholm, and by [18, Chapter 6, the sequence $\left(s_{k}\left(P_{n} A^{*} A P_{n}\right)\right)_{n \in \mathbb{N}}$ tends to zero for any $k$.

Corollary 2.7. Let $A: \mathcal{H} \rightarrow \mathcal{H}$ be a normally solvable operator. The homogeneous equation

$$
A f=0
$$

has a non-trivial solution in $\mathcal{H}$ if and only if the sequence $\left(P_{n} A^{*} A P_{n}\right)$ does not have the 0-splitting property.

Proof. Assume that the homogeneous equation (2.6) has a non-trivial solution. If $\operatorname{dim} \operatorname{ker} A=k, k \in \mathbb{N}$, then the sequence $\left(P_{n} A^{*} A P_{n}\right)$ is subject to the $k$-splitting property with $k>0$. If $\operatorname{dim} \operatorname{ker}\left(I-R_{a}\right)=\infty$, then $I-R_{a}$ is not a $\Phi^{+}$operator and all sequences of singular values converge to zero. Conversely, if the sequence $\left(P_{n} A^{*} A P_{n}\right)$ does not have the 0 -splitting property, then it either has a $k$-splitting property with $k>0$, or all sequences of singular values converge to zero. In the first case, equation (2.6) has $k$ linearly independent solutions. In the second case, $A$ is not a $\Phi^{+}$operator. Since $A$ is supposed to be normally solvable, $\operatorname{dim} \operatorname{ker}\left(I-R_{a}\right)$ has to be infinite.

\section{Fredholm properties of the operator $I-R_{a}$}

In this section, we examine Fredholm properties and the invertibility of the operator $I-R_{a}$ for symbols $a$ whose Fourier series converge uniformly.

For any fixed positive real number $q$, consider an operator $B_{q}: L_{2}(\mathbb{R}) \rightarrow L_{2}(\mathbb{R})$ defined by

$$
B_{q} f(t)=f(q t)
$$

Where there can be no confusion, we drop the index 2 in the case $q=2$, so the corresponding dilation operator $B_{2}$ is simply denoted by $B$. 
Let $f \in L_{2}(\mathbb{R})$, and let $\mathfrak{F}$ and $\mathfrak{F}^{-1}$ denote the direct and inverse Fourier transforms, respectively; namely

$$
\begin{aligned}
(\mathfrak{F} f)(t) & =\frac{1}{\sqrt{2 \pi}} \int_{\mathbb{R}} e^{-i y t} f(y) d y, \\
\left(\mathfrak{F}^{-1} f\right)(t) & =\frac{1}{\sqrt{2 \pi}} \int_{\mathbb{R}} e^{i y t} f(y) d y, \quad t \in \mathbb{R} .
\end{aligned}
$$

If $a \in L_{\infty}(\mathbb{R})$, it is well known that the convolution operator $C(a)=\mathfrak{F} a \mathfrak{F}^{-1}$ is bounded on the space $L_{2}(\mathbb{R})$ and

$$
\left\|\mathfrak{F} a \mathfrak{F}^{-1}\right\|_{2}=\|a\|_{\infty}
$$

We also consider the shift operator $V: L_{2}(\mathbb{R}) \rightarrow L_{2}(\mathbb{R})$ defined by

$$
V f(t)=f(t-1), \quad t \in \mathbb{R} .
$$

It is easily seen that for any polynomial

$$
p_{n}(z)=\sum_{k=-n}^{n} a_{k} z^{k}, \quad z \in \mathbb{T},
$$

the operator $C\left(p_{n}\right)$ is

$$
C\left(p_{n}\right)=p_{n}(V)=\sum_{k=-n}^{n} a_{k} V^{k}
$$

Thus if the Fourier series $\mathcal{S}(a)$ of the function $a$ converges uniformly, then by continuity

$$
C(a)=\lim _{n \rightarrow \infty} \mathcal{S}_{n}(V)
$$

where $\mathcal{S}_{n}(a)$ are the partial sums of $\mathcal{S}(a)$. Hence, if $a \in U C_{\mathcal{F}}(\mathbb{T})$, then the refinement operator

$$
R_{a} f=\sum_{k \in \mathbb{Z}} a_{k} f(2 \cdot-k)
$$

can be represented as a product of the two operators $B$ and $C(a)$, that is,

$$
R_{a}=B C(a) \text {. }
$$

Lemma 3.1. Let $a \in U C_{\mathcal{F}}(\mathbb{T})$. The operator $I-R_{a}: L_{2}(\mathbb{R}) \rightarrow L_{2}(\mathbb{R})$ is a $\Phi^{+}$ operator (invertible) if and only if the operator $G_{a}=I-(a / 2) B_{1 / 2}: L_{2}(\mathbb{R}) \rightarrow L_{2}(\mathbb{R})$ is a $\Phi^{+}$operator (invertible). Moreover,

$$
\operatorname{dim} \operatorname{ker}\left(I-R_{a}\right)=\operatorname{dim} \operatorname{ker} G_{a} .
$$

Proof. Since the operators $B_{2}$ and $\mathfrak{F}$ are invertible in $\mathcal{L}\left(L_{2}(\mathbb{R})\right)$ and

$$
B_{1 / 2} \mathfrak{F}=2 \mathfrak{F} B_{2},
$$

the operator $I-R_{a}$ can be rewritten as

$$
\begin{aligned}
I-R_{a} & =B_{2} \mathfrak{F}\left(\mathfrak{F}^{-1} B_{1 / 2} \mathfrak{F}-a I\right) \mathfrak{F}^{-1} \\
& =2 B_{2} \mathfrak{F}\left(I-\frac{a}{2} B_{1 / 2} I\right) B_{2} \mathfrak{F}^{-1}
\end{aligned}
$$

and the lemma follows.

Lemma 3.2. Let $a \in U C_{\mathcal{F}}(\mathbb{T})$ and let $\widetilde{\Delta}(a)$ be the non-negative number defined by (1.8). If the refinement equation (1.1) has a non-trivial solution, then $\widetilde{\Delta}(a) \geq \sqrt{2}$. Moreover, if in addition, $a(x) \neq 0$ for all $x \in[0,2 \pi]$, then $\sqrt{2} \in\left[\widetilde{\Delta}^{-1}\left(a^{-1}\right), \widetilde{\Delta}(a)\right]$. 
Proof. Assume that equation (1.1) has a non-trivial solution but $\widetilde{\Delta}(a)<\sqrt{2}$. Consider the operator $T_{a}:=(a / 2) B_{1 / 2}$. Since

$$
T_{a}^{n}=B_{1 / 2}^{n} \frac{\prod_{j=1}^{n} a\left(2^{j} \cdot\right)}{2^{n}} I, \quad n \in \mathbb{N},
$$

and

$$
\left\|B_{1 / 2}^{n} f\right\|=2^{n / 2}\|f\|, \quad f \in L_{2}(\mathbb{R}),
$$

the spectral radius $\rho\left(T_{a}\right)$ of the operator $T_{a}$ is

$$
\rho\left(T_{a}\right)=\frac{\widetilde{\Delta}(a)}{\sqrt{2}} .
$$

Thus $\rho\left(T_{a}\right)<1$, and the operator $G_{a}$ is invertible. By Lemma 3.1, equation (1.1) only has the trivial solution, but this contradicts our assumption, so $\widetilde{\Delta}(a) \geq \sqrt{2}$. If $a(x) \neq 0$ on $[0,2 \pi]$, we represent the operator $G_{a}$ in the form

$$
G_{a}=-\frac{a}{2}\left(I-\frac{2}{a} B_{2}\right) B_{1 / 2}
$$

Now one can mention that

$$
\left\|\left(\frac{2}{a} B_{2}\right)^{n}\right\|=\left\|\left(\left(\frac{2}{a} B_{2}\right)^{n}\right)^{*}\right\|=\left\|T_{2 / \bar{a}}^{n}\right\| .
$$

Applying the previous arguments to the operator $T_{2 / \bar{a}}$, we obtain $\widetilde{\Delta}^{-1}\left(a^{-1}\right) \leq \sqrt{2}$. Thus if equation (1.1) has non-trivial solutions and $a$ does not vanish, then $\sqrt{2} \in$ $\left[\widetilde{\Delta}^{-1}\left(a^{-1}\right), \widetilde{\Delta}(a)\right]$.

As a next step, we consider the dimension of the kernel space for the operator $I-R_{a}$.

Theorem 3.3. Let $R_{a}$ be the refinement operator generated by a function a $\in$ $U C_{\mathcal{F}}(\mathbb{T})$. If $I-R_{a} \in \Phi^{+}\left(L_{2}(\mathbb{R})\right)$, then

$$
\operatorname{dim} \operatorname{ker}\left(I-R_{a}\right)=0 .
$$

Proof. To investigate the kernel space of the operator $I-R_{a}$ we again turn to the operator $G_{a}$. Assume that $I-R_{a}$ is a $\Phi^{+}$operator. By Lemma 3.1, the operator $G_{a} \in \Phi^{+}\left(L_{2}(\mathbb{R})\right)$ and $\operatorname{dim} \operatorname{ker} G_{a}=\operatorname{dim} \operatorname{ker}\left(I-R_{a}\right)$. Hence, if the homogeneous refinement equation (1.1) has a non-trivial solution, then there exists an element $f_{0} \in L_{2}(\mathbb{R}), f_{0} \neq 0$ such that

$$
a(x) f_{0}(x / 2)=2 f_{0}(x)
$$

almost everywhere on $\mathbb{R}$. Since $f_{0} \neq 0$, one can find an interval $\mathcal{I} \subset \mathbb{R}^{+}$or an interval $\mathcal{I} \subset \mathbb{R}^{-}$such that

$$
\int_{\mathcal{I}}\left|f_{0}(x)\right|^{2} d x>0 .
$$

Assume for definiteness that $\mathcal{I}$ is in $\mathbb{R}^{+}$, and consider any element $m \in L_{\infty}([1,2))$ with ess $\inf _{x \in[1,2)}|m(x)| \geq \epsilon>0$. From the interval [1,2) this element can first be extended on the intervals $\left[2^{-1}, 1\right)$ and $\left[1,2^{2}\right)$ by

$$
\begin{gathered}
m(x):=m(2 x) \quad \text { if } \quad x \in\left[2^{-1}, 1\right), \\
m(x):=m(x / 2) \quad \text { if } \quad x \in\left[2,2^{2}\right)
\end{gathered}
$$


and then gradually on the whole positive semi-axis $\mathbb{R}^{+}$. (For simplicity we use the same letter $m$ to denote this continuation.) If one now considers the function

$$
\tilde{m}(x)=\left\{\begin{array}{lll}
m(x) & \text { if } & x \in \mathbb{R}^{+}, \\
0 & \text { if } & x \in \mathbb{R}^{-} \cup\{0\},
\end{array}\right.
$$

then

$$
\underset{x \in \mathbb{R}^{+}}{\operatorname{essinf}}|\widetilde{m}(x)| \geq \epsilon>0
$$

and

$$
\widetilde{m}(x)=\widetilde{m}(x / 2), \quad x \in \mathbb{R} .
$$

However, $\widetilde{m} f_{0} \in \operatorname{ker}_{L_{2}(\mathbb{R})} G_{a}$, and $\widetilde{m} f_{0} \neq 0$ because

$$
\left\|\widetilde{m} f_{0}\right\|^{2} \geq \int_{\mathcal{I}}\left|\widetilde{m} f_{0}(x)\right|^{2} d x \geq \epsilon^{2} \int_{\mathcal{I}}\left|f_{0}(x)\right|^{2} d x>0 .
$$

This obviously implies the relation

$$
\operatorname{dim} \operatorname{ker} G_{a}=\infty,
$$

but $G_{a}$ is a $\Phi^{+}$operator, so the dimension of its null space is finite. Hence

$$
\operatorname{dim} \operatorname{ker}\left(I-R_{a}\right)=0,
$$

and the proof is complete.

Note that in the course of the proof we also established a result which is interesting on its own, which we formulate as a corollary.

Corollary 3.4. Let $a \in U C_{\mathcal{F}}(\mathbb{T})$. If the homogeneous equation (1.1) has a nontrivial $L_{2}$-solution, then

$$
\operatorname{dim} \operatorname{ker}_{L_{2}(\mathbb{R})}\left(I-R_{a}\right)=\infty .
$$

The kernel of the adjoint operator can be studied analogously. As a result one has the following corollary from the representation

$$
\left(I-R_{a}\right)^{*}=\left(2 B_{2} \mathfrak{F}\left(I-\frac{a}{2} B_{1 / 2} I\right) B_{2} \mathfrak{F}^{-1}\right)^{*}=\frac{1}{2} \mathfrak{F} B_{1 / 2}\left(I-\bar{a} B_{2}\right) \mathfrak{F}^{-1} B_{1 / 2},
$$

and from our previous considerations.

Corollary 3.5. Let $a \in U C_{\mathcal{F}}(\mathbb{T})$. If $\left(I-R_{a}\right): L_{2}(\mathbb{R}) \mapsto L_{2}(\mathbb{R})$ is a $\Phi^{-}$-operator, then

$$
\operatorname{dim} \operatorname{ker}\left(I-R_{a}\right)^{*}=0 .
$$

Combining Theorem 3.3 and Corollary 3.5, one obtains the following criterion of the Fredholmness for the operator $I-R_{a}$.

Corollary 3.6. Let $a \in U C_{\mathcal{F}}(\mathbb{T})$. The operator $I-R_{a}$ is Fredholm if and only if it is invertible.

Finally, the results of this section are summarized by the next theorem.

Theorem 3.7. Let $a \in U C_{\mathcal{F}}(\mathbb{T})$. The following are true:

(1) If a does not vanish in the interval $[0,2 \pi]$, then:

(a) If $\sqrt{2} \notin\left[\widetilde{\Delta}^{-1}\left(a^{-1}\right), \widetilde{\Delta}(a)\right]$, then $\operatorname{dim} \operatorname{ker}_{L_{2}(\mathbb{R})}\left(I-R_{a}\right)=0$.

(b) If $\sqrt{2} \in\left[\widetilde{\Delta}^{-1}\left(a^{-1}\right), \widetilde{\Delta}(a)\right]$, then $\operatorname{dim} \operatorname{ker}_{L_{2}(\mathbb{R})}\left(I-R_{a}\right)=\infty$ or $\operatorname{dim} \operatorname{ker}_{L_{2}(\mathbb{R})}\left(I-R_{a}\right)=0$. 
(2) If a vanishes in the interval $[0,2 \pi]$, then

(a) If $\widetilde{\Delta}(a)<\sqrt{2}$, then $\operatorname{dim} \operatorname{ker}_{L_{2}(\mathbb{R})}\left(I-R_{a}\right)=0$.

(b) If $\widetilde{\Delta}(a) \geq \sqrt{2}$, then $\operatorname{dim} \operatorname{ker}_{L_{2}(\mathbb{R})}\left(I-R_{a}\right)=\infty$ or $\operatorname{dim} \operatorname{ker}_{L_{2}(\mathbb{R})}\left(I-R_{a}\right)=0$.

\section{Solvability of THE REFinement EQUATION}

For any given $a \in L_{\infty}(\mathbb{R})$, let $A_{a}$ stand for the operator $I-R_{a}$.

Lemma 4.1. Let $a \in U C_{\mathcal{F}}(\mathbb{T})$, and let the sequence $\left\{b_{l}\right\}$ be defined by

$$
b_{l}:=\frac{1}{2} \sum_{j \in \mathbb{Z}} \bar{a}_{j-l} a_{j}, \quad l \in \mathbb{Z} .
$$

Then $A_{a}^{*} A_{a}$ is a difference operator defined by

$$
\begin{aligned}
A_{a}^{*} A_{a} f(t)= & f(t)+\sum_{l \in \mathbb{Z}} b_{l} f(t-l) \\
& -\sum_{l \in \mathbb{Z}} a_{l} f(2 t-l)-\frac{1}{2} \sum_{l \in \mathbb{Z}} \bar{a}_{l} f\left(\frac{t+l}{2}\right), \quad t \in \mathbb{R} .
\end{aligned}
$$

Proof. Using the easily verified relation $B^{*}=(1 / 2) B_{1 / 2}$, given in [13], one obtains

$$
\begin{aligned}
A_{a}^{*} A_{a} & =\left(I-(1 / 2) C(\bar{a}) B_{1 / 2}\right)(I-B C(a)) \\
& =I+(1 / 2) C(\bar{a}) B_{1 / 2} B C(a)-B C(a)-(1 / 2) C(\bar{a}) B_{1 / 2} \\
& =I+(1 / 2) C\left(|a|^{2}\right)-B C(a)-(1 / 2) C(\bar{a}) B_{1 / 2} .
\end{aligned}
$$

It remains to note that $b_{l}, l \in \mathbb{Z}$ are the Fourier coefficients of the function $(1 / 2)|a|^{2}$, and invoke (3.2).

To make use of the results presented in $\oint 2$, let us consider a scaling function $\varphi$ that satisfies the refinement equation

$$
\varphi(t / 2)=\sum_{k \in \mathbb{Z}} p_{k} \varphi(t-k), \quad t \in \mathbb{R},
$$

and generates a multiresolution analysis 22

$$
\{0\} \leftarrow \ldots \subset \mathcal{U}_{-1} \subset \mathcal{U}_{0} \subset \mathcal{U}_{1} \subset \ldots \rightarrow L_{2}(\mathbb{R}) .
$$

Then the family $\left\{\varphi_{k}\right\}, \varphi_{:}=\varphi(\cdot-k)$ constitutes a Riesz basis for $\mathcal{U}_{0}$. Without loss of generality, we can assume that this basis is orthonormal and consider a function $\psi$ defined by

$$
\psi(t):=\sum_{k}(-1)^{k} p_{1-k} \varphi(2 t-k), \quad t \in \mathbb{R} .
$$

Let $\psi_{s, k}, s, k \in \mathbb{Z}$ denote the functions

$$
\psi_{s, k}(t):=2^{s / 2} \psi\left(2^{s} t-k\right), \quad s, k \in \mathbb{Z} .
$$

Then the space $L_{2}(\mathbb{R})$ can be represented as a direct sum of orthogonal subspaces

$$
L_{2}(\mathbb{R})=\mathcal{U} \oplus \mathcal{W}_{0} \oplus \mathcal{W}_{1} \oplus \ldots \oplus \mathcal{W}_{j} \oplus \ldots,
$$

where

$$
\mathcal{U}=\mathcal{U}_{0}:=\operatorname{clos}_{\operatorname{span}}\left\{\varphi_{L_{2}}: k \in \mathbb{Z}\right\}
$$


and

$$
\mathcal{W}_{s}:=\operatorname{clos} \operatorname{span}_{L_{2}}\left\{\psi_{s, k}: k \in \mathbb{Z}\right\}, \quad s \in \mathbb{Z}^{+} .
$$

We also recall the decomposition

$$
\mathcal{U}_{1}=\mathcal{U}_{0} \oplus \mathcal{W}_{0}
$$

of the subspace $\mathcal{U}_{1}$ into a direct sum of two orthogonal subspaces, and the corresponding "decomposition relation"

$$
\varphi(2 t-i)=\frac{1}{2} \sum_{l \in \mathbb{Z}}\left\{p_{i-2 l} \varphi(t-l)+(-1)^{i} p_{2 l-i+1} \psi(t-l)\right\}, \quad i \in \mathbb{Z},
$$

which holds for any $x \in \mathbb{R}$; cf. [5, p. 63].

According to representation (4.6), let us introduce a linear space $X$ by

$$
X:=\mathcal{U} \times \mathcal{W}_{0} \times \ldots \times \mathcal{W}_{s} \times \ldots
$$

and provide it with the norm

$$
\|x\|:=\left(\|u\|^{2}+\sum_{s \in \mathbb{Z}^{+}}\left\|w_{s}\right\|^{2}\right)^{1 / 2}
$$

where

$$
x=\left(u, w_{0}, w_{1}, \ldots\right)^{T}, \quad u \in \mathcal{U}, \quad w_{s} \in \mathcal{W}_{s}, \quad s \in \mathbb{Z}^{+} .
$$

For a subspace $Y \subset L_{2}(\mathbb{R})$, let $P_{Y}$ denote the orthogonal projection onto $Y$. Consider now a mapping $\eta: L_{2}(\mathbb{R}) \rightarrow X$ defined by

$$
\eta(f):=\left(P_{\mathcal{U}} f, P_{\mathcal{W}_{0}} f, P_{\mathcal{W}_{1}} f, \ldots\right)^{T}
$$

It is easily seen that the mapping $\eta: L_{2}(\mathbb{R}) \rightarrow X$ is a continuously invertible linear operator, and if $g=\left(u, w_{0}, w_{1}, \ldots\right)^{T} \in X$, then

$$
\eta^{-1} g=u+\sum_{s \in \mathbb{Z}^{+}} w_{s}
$$

Moreover, the mapping $\Lambda_{\eta}: \mathfrak{L}\left(L_{2}(\mathbb{R})\right) \rightarrow \mathfrak{L}(X)$ defined by

$$
\Lambda_{\eta}(K)=\eta K \eta^{-1}, \quad K \in \mathfrak{L}
$$

is an isometrical isomorphism. Hence an operator $K \in \mathfrak{L}\left(L_{2}(\mathbb{R})\right)$ is a $\Phi^{+}$or $\Phi^{-}$ operator if and only if $\Lambda_{\eta}(K) \in \mathfrak{L}(X)$ is a $\Phi^{+}$or $\Phi^{-}$operator, respectively. In addition, it follows from (4.8) that if $K \in \Phi^{+}$, then

$$
\operatorname{dim} \operatorname{ker} K=\operatorname{dim} \operatorname{ker} \Lambda_{\eta}(K) .
$$

Thus one can study the Fredholm properties of the operator $K$ by means of the operator $\Lambda_{\eta}(K)$. In many cases, the last operator can be handled with less effort than the operator $K$.

The following lemma describes the structure of the operator $\Lambda_{\eta}(K)$.

Lemma 4.2. Let $K \in \mathfrak{L}$, and let $\Lambda_{\eta}$ be the mapping defined in (4.8). Then

$$
\Lambda_{\eta}(K)=\left(\begin{array}{cccc}
P_{\mathcal{U}} K P_{\mathcal{U}} & P_{\mathcal{U}} K P_{\mathcal{W}_{0}} & P_{\mathcal{U}} K P_{\mathcal{W}_{1}} & \cdots \\
P_{\mathcal{W}_{0}} K P_{\mathcal{U}} & P_{\mathcal{W}_{0}} K P_{\mathcal{W}_{0}} & P_{\mathcal{W}_{0}} K P_{\mathcal{W}_{1}} & \ldots \\
P_{\mathcal{W}_{1}} K P_{\mathcal{U}} & P_{\mathcal{W}_{1}} K P_{\mathcal{W}_{0}} & P_{\mathcal{W}_{1}} K P_{\mathcal{W}_{1}} & \cdots \\
\vdots & \vdots & \vdots & \ddots
\end{array}\right) .
$$


The proof of this result follows immediately from (4.6) and from the definition of the operator $\Lambda_{\eta}$.

Now let us introduce finite-dimensional operators $P_{n m}, n \in \mathbb{Z}, m \in \mathbb{Z}^{+}$defined on the space $L_{2}(\mathbb{R})$ by

$$
P_{n m} f:=\sum_{k=-n}^{n} f_{k} \varphi_{k}+\sum_{s=0}^{m} \sum_{k=-n}^{n} f_{s k} \psi_{s, k},
$$

where $f_{k}:=\left(f, \varphi_{k}\right), k \in \mathbb{Z}$ and $f_{s k}:=\left(f, \psi_{s, k}\right), s \in \mathbb{Z}^{+}, k \in \mathbb{Z}$. It follows from (4.4) that the sequence $\left\{P_{n m}\right\}$ strongly converges to the identity operator as $n$ and $m$ tend to $\infty$. Moreover, it is worth noting that

$$
P_{n m} P_{\mathcal{W}_{s}}=0, \quad P_{\mathcal{W}_{s}} P_{n m}=0, \quad \text { for any } s>m .
$$

To apply the results of $₫ 2$, we identify the matrices of the finite-dimensional operators which appear after applying the Galerkin method based on the projections $P_{n m}$ to the operators from (4.2). Note that for basic aspects of the Galerkin method the reader may consult [15] or [18].

Let $A$ be a linear bounded operator, which acts from a separable Hilbert space $\mathcal{H}_{1}$ to a separable Hilbert space $\mathcal{H}_{2}$; let $\left\{e_{k}\right\}_{k \in \mathbb{Z}}$ and $\left\{\widetilde{e}_{k}\right\}_{k \in \mathbb{Z}}$ be orthonormal bases of $\mathcal{H}_{1}$ and $\mathcal{H}_{2}$, respectively. Introduce the matrix

$$
\widetilde{A}:=\left(a_{j k}\right)_{j, k \in \mathbb{Z}}
$$

where

$$
a_{j k}=\left(A e_{j}, \widetilde{e}_{k}\right)
$$

It is well known that the operator $A$ can be identified with the matrix

$$
\mathbf{A}:=\widetilde{A}^{T} \text {. }
$$

In the following, such an identification is used without any additional comment.

For any operator $K \in \mathfrak{L}\left(L_{2}(\mathbb{R})\right)$, let $K_{n m}$ denote the operator $P_{n m} K P_{n m}$. Then, taking into account (4.12) one obtains

$$
\begin{aligned}
& \Lambda_{\eta}\left(K_{n m}\right)= \\
& \left(\begin{array}{cccccc}
P_{\mathcal{U}} K_{n m} P_{\mathcal{U}} & P_{\mathcal{U}} K_{n m} P_{\mathcal{W}_{0}} & \ldots & P_{\mathcal{U}} K_{n m} P_{\mathcal{W}_{m}} & 0 & \ldots \\
P_{\mathcal{W}_{0}} K_{n m} P_{\mathcal{U}} & P_{\mathcal{W}_{0}} K_{n m} P_{\mathcal{W}_{0}} & \ldots & P_{\mathcal{W}_{0}} K_{n m} P_{\mathcal{W}_{m}} & 0 & \ldots \\
\vdots & \vdots & \vdots & \vdots & \vdots & \ldots \\
P_{\mathcal{W}_{m}} K_{n m} P_{\mathcal{U}} & P_{\mathcal{W}_{m}} K_{n m} P_{\mathcal{W}_{0}} & \ldots & P_{\mathcal{W}_{m}} K_{n m} P_{\mathcal{W}_{m}} & 0 & \ldots \\
0 & 0 & 0 & 0 & 0 & \ldots \\
\vdots & \vdots & \vdots & \vdots & \vdots & \ddots
\end{array}\right)
\end{aligned}
$$

Hence $\Lambda_{\eta}\left(K_{n m}\right)$ is the block matrix operator

$$
\Lambda_{\eta}\left(K_{n m}\right)=\left(A_{r, s}\right)_{r, s=-1}^{m}=\left(P_{r} K_{n m} P_{s}\right)_{r, s=-1}^{m},
$$

where for convenience we set $P_{s}:=P_{W_{s}}$ if $s=0,1, \ldots, m$ and $P_{-1}:=P_{V}$. 
Now consider the structure of the corresponding matrix (4.14) for each of the four operators

$$
\begin{aligned}
I f(t) & =f(t), \\
T^{(1)} f(t) & =\sum_{l \in \mathbb{Z}} b_{l} f(t-l), \\
T^{(2)} f(t) & =-\sum_{l \in \mathbb{Z}} a_{l} f(2 t-l), \\
T^{(3)} f(t) & =-\frac{1}{2} \sum_{l \in \mathbb{Z}} \bar{a}_{l} f\left(\frac{t+l}{2}\right)
\end{aligned}
$$

that appear on the left-hand side of (4.2), and proceed with the description of the blocks $A_{r s}, r, s=-1,0, \ldots, m$ that make up matrices (4.15) for the operators $I$ and $T^{(i)}, i=1,2,3$.

For any given $n \in \mathbb{N}$, let $I_{n}$ denote the identity $(2 n+1) \times(2 n+1)$ matrix, i.e.,

$$
I_{n}:=\left(\delta_{j k}\right)_{k, j=-n}^{n} .
$$

Then

$$
\mathbf{I}_{n, m}=\Lambda_{\eta}\left(I_{n m}\right)=\operatorname{diag}(\underbrace{I_{n}, \ldots, I_{n}}_{m+1 \text { times }}) .
$$

Recall that for any fixed $l \in \mathbb{Z}$, the shift operator $V^{l}$ is defined by

$$
V^{l} f:=f(\cdot-l), \quad l \in \mathbb{Z} .
$$

It is easily seen that

$$
V^{l} \varphi_{j}=\varphi_{j+l}, \quad j, l \in \mathbb{Z}
$$

and

$$
V^{l} \psi_{s, j}=\psi_{s, 2^{s} l+j}, \quad s \in \mathbb{Z}^{+}, \quad j, l \in \mathbb{Z} .
$$

Hence all subspaces $\mathcal{U}, \mathcal{W}_{0}, \mathcal{W}_{1}, \ldots$ are invariant with respect to the operator $T^{(1)}$, and the operator $\Lambda_{\eta}\left(T_{n m}^{(1)}\right)$ can be identified with the block diagonal matrix

$$
\mathbf{T}_{n m}^{(1)}:=\left(\delta_{s r} \mathbf{A}_{s r}^{(1)}\right)_{s, r=-1}^{m},
$$

where

$$
\mathbf{A}_{-1,-1}^{(1)}=\mathbf{A}_{0,0}^{(1)}=\left(b_{k-j}\right)_{k, j=-n}^{n}
$$

and

$$
\mathbf{A}_{s, s}^{(1)}=\left(\sum_{l \in \mathbb{Z}} b_{l} \delta_{2^{s} l+j, k}\right)_{k, j=-n}^{n}
$$

if $s \geq 1$.

Considering the operators

$$
\mathbf{T}_{n m}^{(2)}=\Lambda_{\eta}\left(T_{n m}^{(2)}\right),
$$

let us first mention a property of the basis functions $\psi_{s, j}$ of the spaces $\mathcal{W}_{s}, s \in \mathbb{Z}^{+}$, that is,

$$
\psi_{s, j}(2 t-l)=2^{-1 / 2} \psi_{s+1,2^{s} l+j}, \quad s \in \mathbb{Z}^{+}, \quad j, l \in \mathbb{Z} .
$$

Hence for any $s \in \mathbb{Z}^{+}$and for any $r$ such that $r \neq s+1$ one has

$$
P_{r} P_{n m} T^{(2)} P_{n m} P_{s}=0
$$


and

$$
P_{-1} P_{n m} T^{(2)} P_{n m} P_{s}=0 .
$$

For $r=s+1, s=0,1, \ldots, m-1$, the corresponding matrix representation of the operators $A_{s+1, s}^{(2)}=P_{s+1} P_{n m} T^{(2)} P_{n m} P_{s}$ is (cf. (4.21) $)$

$$
\mathbf{A}_{s+1, s}^{(2)}=\left(-\sum_{l \in \mathbb{Z}} 2^{-1 / 2} a_{l} \delta_{2^{s} l+j, k}\right)_{k, j=-n}^{n} .
$$

The operators $P_{r} P_{n m} T^{(2)} P_{n m} P_{-1}, r=-1,0, \ldots, m$ require separate consideration. Let us start with the operator $P_{-1} P_{n m} T^{(2)} P_{n m} P_{-1}$. Taking into account relation (4.7), one obtains

$$
P_{-1} P_{n m} T^{(2)} P_{n m} P_{-1} \varphi_{j}=-\frac{1}{2} \sum_{l=-n}^{n}\left(\sum_{i \in \mathbb{Z}} a_{i-j} p_{i-2 l}\right) \varphi_{l}, \quad j \in \mathbb{Z}
$$

and hence

$$
\left(P_{-1} P_{n m} T^{(2)} P_{n m} P_{-1} \varphi_{j}, \varphi_{k}\right)=-\frac{1}{2} \sum_{i \in \mathbb{Z}} a_{i-j} p_{i-2 k} .
$$

Thus the operator $P_{-1} P_{n m} T^{(2)} P_{n m} P_{-1}$ can be identified with the matrix

$$
\mathbf{A}_{-1,-1}^{(2)}:=\left(-\frac{1}{2} \sum_{i \in \mathbb{Z}} a_{i-j} p_{i-2 k}\right)_{k, j=-n}^{n} .
$$

For identification of the operator $P_{0} P_{n m} T^{(2)} P_{n m} P_{-1}$, one can use relation (4.7) again. As previously, one obtains

$$
P_{0} P_{n m} T^{(2)} P_{n m} P_{-1} \varphi_{j}=\frac{1}{2} \sum_{l=-n}^{n}\left(\sum_{i \in \mathbb{Z}}(-1)^{i+1} a_{i-j} p_{2 l-i+1}\right) \psi_{l},
$$

which implies

$$
\mathbf{A}_{0,-1}^{(2)}:=\left(\frac{1}{2} \sum_{i \in \mathbb{Z}}(-1)^{i+1} a_{i-j} p_{2 k-i+1}\right)_{k, j=-n}^{n} .
$$

It remains to note that for $r \geq 1$ all $P_{r} P_{n m} T^{(2)} P_{n m} P_{-1}$ are zero operators, so all entries of the corresponding matrices $\mathbf{A}_{r,-1}^{(2)}, r \geq 1$ are equal to 0 .

Consider now the matrix representation for the remaining operator

$$
T^{(3)} f(t)=-\frac{1}{2} \sum_{l \in \mathbb{Z}} f\left(\frac{t+l}{2}\right), \quad t \in \mathbb{R} .
$$

Let us start with the blocks which correspond to the operators $P_{r} P_{n m} T^{(3)} P_{n m} P_{s}$ for $s \geq 1$. First of all, we note that

$$
\psi_{s, j}\left(\frac{t+l}{2}\right)=2^{1 / 2} \psi_{s-1, j-2^{s-1} l}, \quad j, l \in \mathbb{Z}, \quad s \geq 1,
$$

so

$$
P_{r} P_{n m} T^{(3)} P_{n m} P_{s}=0 \quad \text { if } \quad r \neq s-1 .
$$


For $r=s-1$ and $j \in \mathbb{Z}$ one has

$$
P_{s-1} T^{(3)} P_{n m} P_{s} \psi_{s, j}=-\frac{1}{2} \sum_{l \in \mathbb{Z}} 2^{1 / 2} \bar{a}_{l} \psi_{s-1, j-2^{s-1} l},
$$

and therefore

$$
\mathbf{A}_{s-1, s}^{(3)}=\left(-2^{-1 / 2} \sum_{l \in \mathbb{Z}} \bar{a}_{l} \delta_{j-2^{s-1} l, k}\right)_{k, j=-n}^{n} .
$$

The cases $s=-1$ and $s=0$ can be handled by using the refinement equation (4.3) and relation (4.5), which defines the wavelet $\psi$. More precisely, since $\varphi$ satisfies (4.3) one can write

$$
\varphi\left(\frac{t+l}{2}\right)=\sum_{i \in \mathbb{Z}} p_{i} \varphi(t-(i-l)),
$$

so using the definition of $\varphi_{j}, j \in \mathbb{Z}$, one obtains

$$
\varphi_{j}\left(\frac{t+l}{2}\right)=\sum_{i \in \mathbb{Z}} p_{i} \varphi_{i-l+2 j}(t)
$$

and hence

$$
P_{r} P_{n m} T^{(3)} P_{n m} P_{-1}=0 \quad \text { if } \quad r \neq-1 .
$$

Moreover, since

$$
P_{-1} P_{n m} T^{(3)} P_{n m} P_{-1} \varphi_{j}=-\frac{1}{2} \sum_{i=-n}^{n} \sum_{l \in \mathbb{Z}} \bar{a}_{l} p_{i+l-2 j} \varphi_{i}
$$

one gets

$$
\mathbf{A}_{-1,-1}^{(3)}=\left(-\frac{1}{2} \sum_{l \in \mathbb{Z}} \bar{a}_{l} p_{k+l-2 j}\right)_{k, j=-n}^{n} .
$$

This gives a complete description for the representations of the corresponding operators for $s=-1$.

The same approach can be applied to study the case $s=0$, except that relation (4.5) is used instead of (4.3). Repeating the previous arguments leads to the equality

$$
\psi_{0, j}\left(\frac{t+l}{2}\right)=\sum_{i \in \mathbb{Z}}(-1)^{i+l} p_{2 j+1-i-l} \varphi_{i},
$$

whence

$$
P_{r} P_{n m} T^{(3)} P_{n m} P_{0}=0 \quad \text { if } \quad r \neq-1
$$

and

$$
P_{-1} P_{n m} T^{(3)} P_{n m} P_{0} \psi_{j}=\frac{1}{2} \sum_{i=-n}^{n} \sum_{l \in \mathbb{Z}}(-1)^{i+l+1} \bar{a}_{l} p_{2 j+1-i-l} \varphi_{i}
$$

so that

$$
\mathbf{A}_{-1,0}^{(3)}=\left(\frac{1}{2} \sum_{l \in \mathbb{Z}}(-1)^{k+l+1} \bar{a}_{l} p_{2 j+1-k-l}\right)_{k, j=-n}^{n} .
$$


Combining all previous representations, one obtains that the operator $R_{n m}=$ $\Lambda_{\eta}\left(P_{n m} A_{a}^{*} A_{a} P_{n m}\right)$ has a three-diagonal block matrix $\mathbf{R}_{n m}$ given by

$$
\left(\begin{array}{cccccc}
\mathbf{I}_{n}+\sum_{i=1}^{3} \mathbf{A}_{-1,-1}^{(i)} & \mathbf{A}_{-1,0}^{(3)} & 0 & 0 & \ldots & 0 \\
\mathbf{A}_{0,-1}^{(2)} & \mathbf{I}_{n}+\mathbf{A}_{0,0}^{(1)} & \mathbf{A}_{0,1}^{(3)} & \ddots & \ddots & \vdots \\
0 & \mathbf{A}_{1,0}^{(2)} & \mathbf{I}_{n}+\mathbf{A}_{1,1}^{(1)} & \ddots & \ddots & \vdots \\
\vdots & \ddots & \ddots & \ddots & \ddots & 0 \\
\vdots & \ddots & \ddots & \ddots & \mathbf{I}_{n}+\mathbf{A}_{m-1, m-1}^{(1)} & \mathbf{A}_{m-1, m}^{(3)} \\
0 & \ldots & \ldots & 0 & \mathbf{A}_{m, m-1}^{(2)} & \mathbf{I}_{n}+\mathbf{A}_{m, m}^{(1)}
\end{array}\right),
$$

where the structure of the corresponding non-zero matrix blocks $A_{r, s}^{(i)}$ has been established in (4.18), (4.19), (4.24), (4.25), (4.26), (4.28), (4.30), (4.33).

Now the application of Theorems 2.6 and 3.3 and Corollary 2.7 to the sequence $\left(\mathbf{R}_{n m}\right)$ implies the following result.

Theorem 4.3. Let the symbol a of the refinement operator $R_{a}$ have uniformly convergent Fourier series and satisfy the condition

$$
\widetilde{\Delta}(a) \geq \sqrt{2}
$$

if a vanishes in the interval $[0,2 \pi]$, or the condition

$$
\sqrt{2} \in\left[\widetilde{\Delta}^{-1}\left(a^{-1}\right), \widetilde{\Delta}(a)\right]
$$

if a does not vanish in the interval $[0,2 \pi]$.

Then

(1) If the refinement equation (1.1) is solvable, then all sequences of singular values of the matrices (4.34) converge to zero.

(2) If the operator $I-R_{a}$ is normally solvable, then the homogeneous refinement equation (1.1) has a non-trivial solution in the space $L_{2}(\mathbb{R})$ if and only if all sequences of singular values of matrices (4.34) tend to zero as $n \rightarrow \infty$ and $m \rightarrow \infty$.

Corollary 4.4. Let the conditions of Theorem 4.3 be satisfied. If $I-R_{a}$ is a normally solvable operator, then the refinement equation (1.1) has a non-trivial $L_{2}$ solution if and only if the sequence of the smallest singular values of the matrices $\mathbf{R}_{n m}$ converges to zero.

The solvability of the non-homogeneous refinement equation $(1.9)$ in $L_{2}(\mathbb{R})$ can also be characterized.

Theorem 4.5. Let $a \in U C_{\mathcal{F}}(\mathbb{T})$. The non-homogeneous refinement equation (1.9) is solvable for any right-hand side $g \in L_{2}(\mathbb{R})$ and the solution is unique if and only if there are $m_{0}, n_{0} \in \mathbb{N}$ such that the set $\left\{s_{n m}(1): n \geq n_{0}, m \geq m_{0}\right\}$ which comprises the smallest singular values of the matrices $\mathbf{R}_{n m}$ of (4.34) is bounded away from zero. 
Remark 4.6. In view of Lemma 1.1. one can also adopt the operators $G_{a}=I-$ $(a / 2) B_{1 / 2}$ to investigate the solvability of the refinement equations (1.1) and (1.9). Then the Galerkin approximations of the operator

$$
G_{a}^{*} G_{a}=I+\frac{|a|^{2}}{2} I-\frac{a}{2} B_{1 / 2}-\bar{a} B_{2}
$$

have to be studied. However, although the operator $G_{a}^{*} G_{a}$ appears simpler than the operator $A^{*} A$ of (4.2), the last one is more suitable for approximation via wavelet bases. As far as the operator $G_{a}^{*} G_{a}$ is concerned, it is not entirely clear what kind of basis system could be applied to obtain matrices as well structured as $\mathbf{R}_{n m}$.

\section{Numerical tests: Solvability in $L_{2}(\mathbb{R})$}

To apply the preceding results to specific refinement equations, one must be able to evaluate sequences of singular values of matrices $\mathbf{R}_{n m}$. Let us denote the sequence of the smallest singular values of these matrices by $\left\{s_{n m}(1)\right\}$, the sequence of the second smallest singular values by $\left\{s_{n m}(2)\right\}$, and so on. Although the matrices $\mathbf{R}_{n m}$ of (4.34) have a nice structure, the analytic estimate of their singular values seems to be extremely difficult, even for the refinement equations possessing a minimal number of non-zero coefficients. In this situation, a numerical approach to obtain the singular values $s_{n m}(k), k \in \mathbb{N}$ can be helpful. However, the convergence of such sequences can be slow, which may prevent evidence of the effect mentioned in Theorem 4.3. Indeed, since the matrix $\mathbf{R}_{n m}$ has $(m+2)(2 n+1)$ rows and $(m+2)(2 n+1)$ columns, it rapidly becomes unmanageable as $m$ and $n$ increase. Nevertheless, for certain classes of refinement operators one can handle matrices $\mathbf{R}_{n m}$ by using a standard PC and standard MATLAB tools for the computation of singular values. Of course, more powerful computers or more sophisticated software could achieve better results.

Let us now call equation (1.1) the test equation, and equation (4.3) that generates the orthonormal wavelet basis the basic equation. In this paper, three equations are chosen as test equations. The vectors of the non-zero coefficients for these equations are:

(I) $[1,1]$;

(II) $[k, k], k \in \mathbb{R}$;

(III) $[3 / 5,6 / 5,2 / 5,-1 / 5]$.

In cases (I) and (III), each equation produces an orthonormal wavelet basis, so the above numbers can be used as the coefficients $a_{k}, k \in \mathbb{Z}$ in the test equations, and/or as the coefficients $p_{k}, k \in \mathbb{Z}$ in the basic equations. In particular, the refinement equation with the coefficients from (I) produces the Haar wavelet [2, 5, 9. In the following this equation is denoted by HW. The refinement equation with the coefficients from (III) produces one of the Daubechies orthogonal wavelets [9]. It will be considered in Section 7. In the case (II), the situation depends on the value of the parameter $k \in \mathbb{R}$ and will be discussed later. Note that the Haar equation is used as a basic equation in all examples presented here. However, other basic refinement equations were also tested, and it is worth noting that the sequences of singular values for the corresponding matrices $\mathbf{R}_{n m}$ demonstrate similar behaviour.

Let us briefly explain the meaning of the symbols used in the figures here and subsequently. In this section the symbol "o" is used for any singular value of the matrix $\mathbf{R}_{n m}$ that belongs to the relevant graph. For any $\alpha \in \mathbb{R}$, the notation 
"ceil $(\alpha)$ " means the smallest integer $m$ such that $\alpha \leq m$. The title for each graph shows which basic equation is used to obtain the picture given, the connection between $n$ and $m$, and the maximal number $n$ used. The numbers on the top of each graph represent the smallest $s(1)$ and second smallest $s(2)$ singular values of the matrix $\mathbf{R}_{n m}$, computed for the maximal values of $n$ and $m$. On the $X$-axis we note the values of the parameter $n$, whereas the $Y$-axis is reserved for the singular values $s_{n m}$ of the corresponding matrices $\mathbf{R}_{n m}$. For the graphs presented in Section 7 we use the cross "x" for the smallest singular value and the dot "•" for the second smallest singular value, whereas the circle "o" is used for any other singular value of $\mathbf{R}_{n m}$ that appears in the designated coordinate window of the corresponding figure. On the other hand, in the case of a 0 -splitting the only relevant sequence is $s_{n}(1)$, so in such a situation we do not highlight the sequence $s_{n}(2)$.

We start with the Haar wavelet equation. The graph in Figure 1 shows the distribution of singular values of the matrices $\mathbf{R}_{n m}$. The computations start from $n=1$ and on each next step the parameter $n$ is increased by 5 . Recall that for any refinement equation the sequences of singular values can exhibit only one of two different behaviours: either they all are bounded away from zero (if there is no solution) or they all converge to zero. As one can see in Figure 1, for the Haar refinement equation the sequence of the smallest singular values becomes extremely small. Other sequences also tend to zero, although the convergence rates are different for different sequences. In particular, they are visibly slower for the sequences $\left\{s_{n m}(k)\right\}$ with $k \geq 7$.

Now consider the equation

$$
f(x)=k f(2 x)+k f(2 x-1), \quad k \in \mathbb{R}
$$

for different values $k \in \mathbb{R}$. As already mentioned, if $k \in(1 / \sqrt{2}, \sqrt{2})$, then this equation has non-trivial solutions in the space $L_{2}(\mathbb{R}),[23$. For $k \neq 1$, all these solutions are not compactly supported, so they remain virtually invisible for the existing methods connected with $L_{1}$-solvability theory.

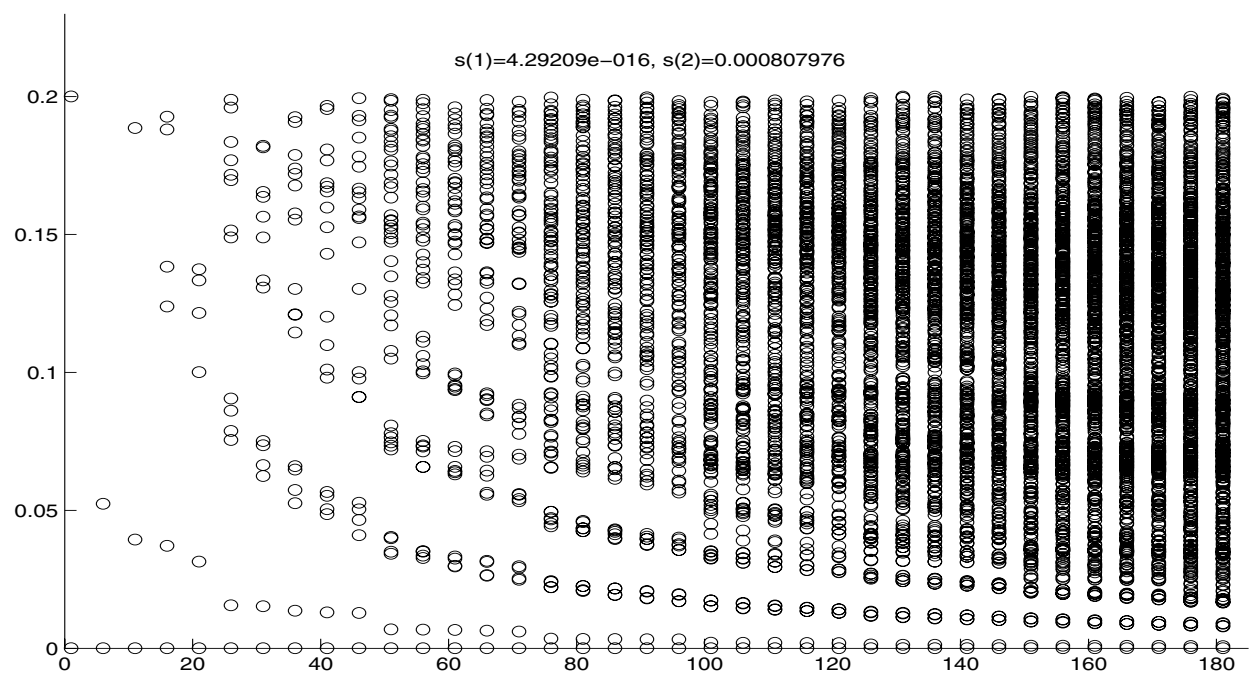

Figure 1. Test Equation: HW; $m=\operatorname{ceil}(n / 25), n_{\max }=181$. 
Note that for equation (5.1) the symbol of the corresponding refinement operator is $a(t)=k+k t, t \in \mathbb{T}$, when $\widetilde{\Delta}(a)=2 k$. Thus, if $k \in(0,1 / \sqrt{2})$, by Theorem 3.7 . equation (5.1) has only the trivial solution in $L_{2}(\mathbb{R})$. Let us check how these results are reflected in the behaviour of singular values of the corresponding matrices. First we take a parameter $k=0.9$ that belongs to the interval $(1 / \sqrt{2}, \sqrt{2})$ and is close to the parameter $k=1$ associated with the Haar refinement equation. Then the distribution of the corresponding singular values is shown in Figure 2, Comparing the graphs in Figures 1 and 2, one observes certain similarities. Although the sequence of the smallest singular values now consists of non-zero elements, it again converges to 0 , in agreement with previous considerations.

However, the situation becomes more ambiguous if one starts with marginal values of the parameter $k$. Figure 3 and Figure 4 below represent situations where equation (5.1) is solvable (for $k=0.75$, which is still in the interval $(1 / \sqrt{2}, \sqrt{2})$ ), or has only the trivial solution (for $k=0.5$, which is in the interval $(0,1 / \sqrt{2})$ ).

It is difficult to decide from Figure 3 and Figure 4 which of these two cases might represent an example of a solvable refinement equation. (To trace the sequence of the lowest singular values in such cases, it is desirable to use a more powerful computer and/or more sophisticated software.) Nevertheless, the above examples show that the results of Theorem 4.3 are not only of theoretical interest, but in some cases give an insight into the solvability of refinement equations in the space $L_{2}(\mathbb{R})$.

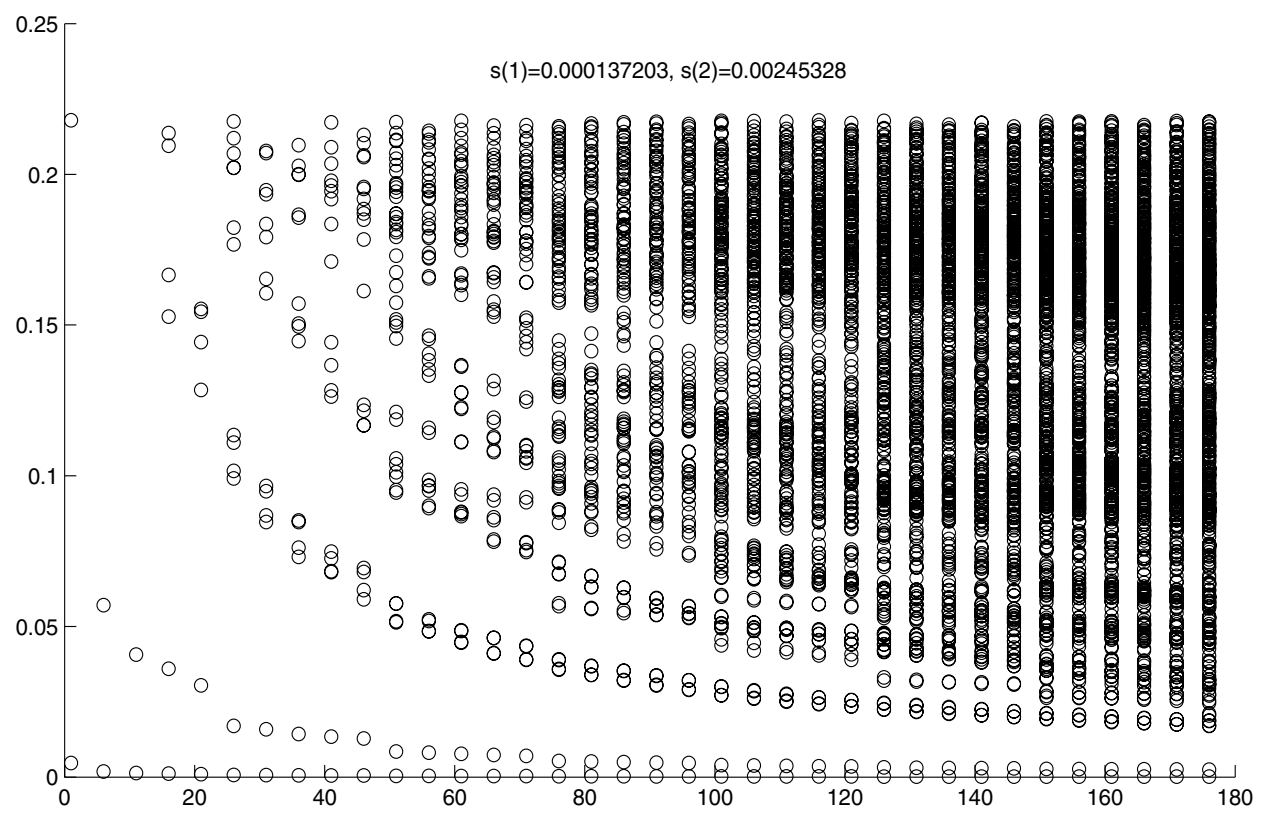

Figure 2. Test Equation: Eqn. (5.1), $k=0.9 ; m=\operatorname{ceil}(n / 25), n_{\max }=176$. 


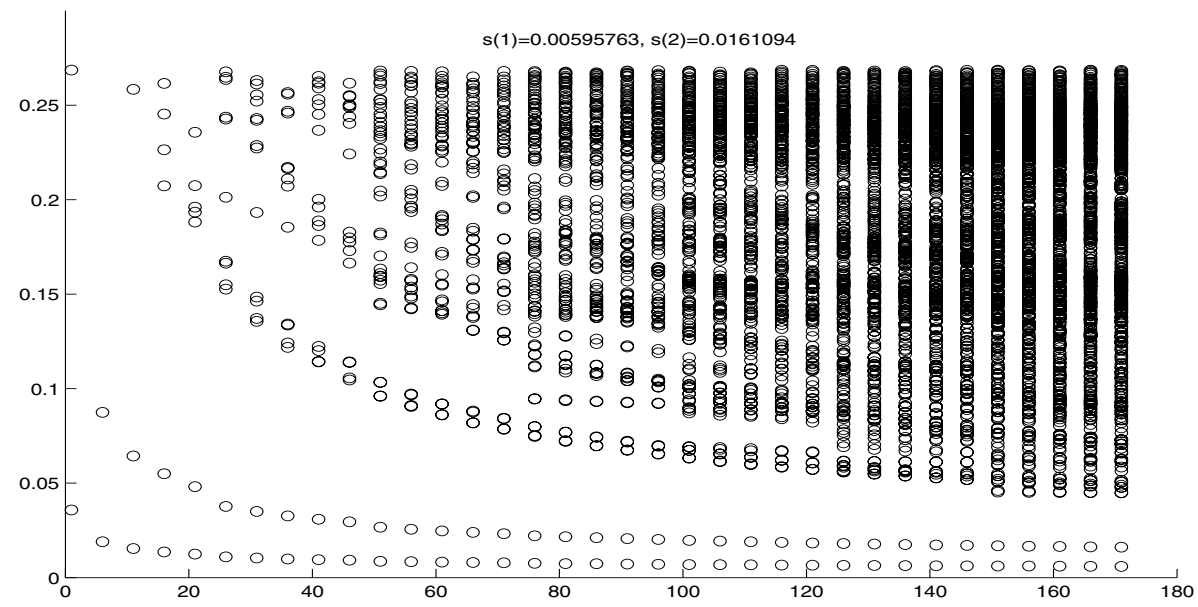

Figure 3. Test Equation: Eqn. (5.1), $k=0.75 ; m=\operatorname{ceil}(n / 25), n_{\max }=171$.

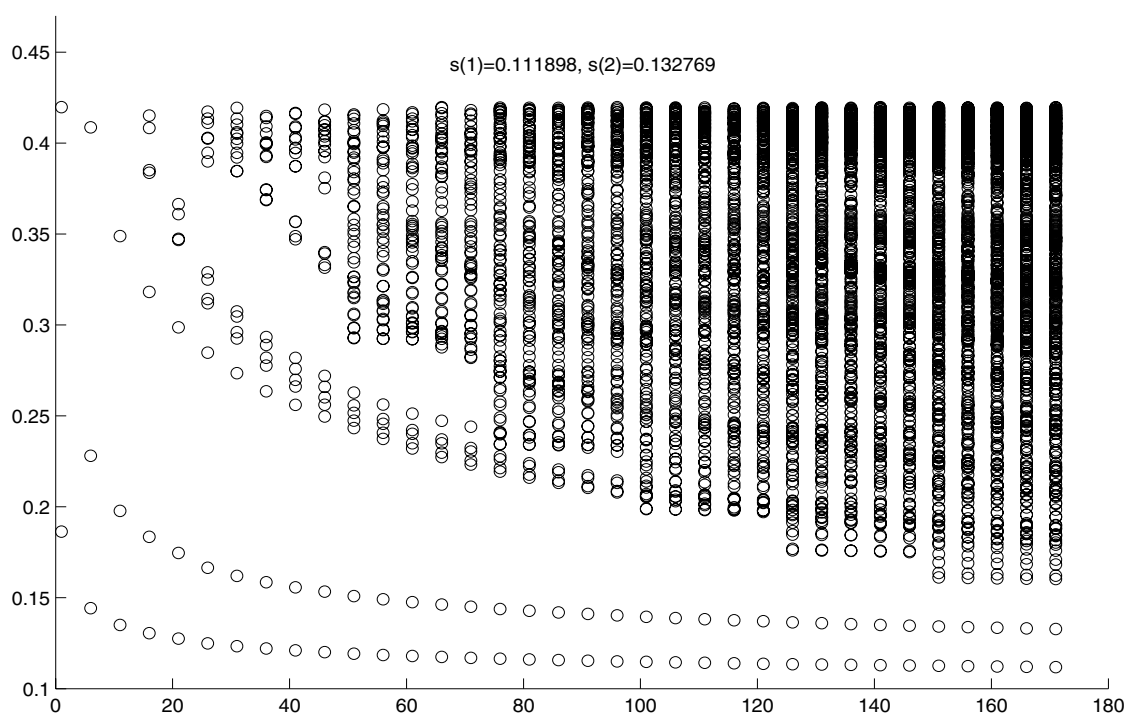

Figure 4. Test Equation: Eqn. (5.1), $k=0.5 ; m=\operatorname{ceil}(n / 25), n_{\max }=176$.

\section{Compactly supported solutions}

For any refinement equation with polynomial symbols

$$
f(t)=\sum_{k=0}^{N} a_{k} f(2 t-k), \quad a_{0} \neq 0, a_{N} \neq 0
$$

one can note that if there is a compactly supported solution $f_{0} \in L_{2}(\mathbb{R})$, then $f_{0} \in$ $L_{1}(\mathbb{R})$, so the properties of such solutions can be described within the framework of $L_{1}$-theory. In particular, it is known that $\operatorname{supp}\left\{f_{0}\right\} \subseteq[0, N], \operatorname{supp}\left\{\left(I-R_{a}\right) f_{0}\right\} \subseteq$ 
$[0, N]$, and the dimension of the space of compactly supported $L_{1}$-solutions of (6.1) is at most one [10]. Thus in the present situation the approach of $₫ 2$ leads to results that differ from those obtained in $\$ \$ 4$.

For any interval $[0, N]$, let us identify the subspace of $L_{2}(\mathbb{R})$ that consists of all elements $f \in L_{2}(\mathbb{R})$ having their support in $[0, N]$ with the space $L_{2}([0, N])$. By $\mathcal{P}_{N}$ we denote the projection operator, $\mathcal{P}_{N}: L_{2}(\mathbb{R}) \mapsto L_{2}([0, N])$ defined by

$$
\mathcal{P}_{N} f(t)=\left\{\begin{array}{cl}
f(t) & \text { if } t \in[0, N], \\
0 & \text { otherwise. }
\end{array}\right.
$$

Using this projection, one can rewrite equation (6.1) considered on the space $L_{2}([0, N])$ as

$$
\mathcal{P}_{N}\left(I-R_{a}\right) \mathcal{P}_{N} f=0 .
$$

Thus to investigate the solvability of (6.1) in the space of compactly supported $L_{2}$-functions, one has to study the behavior of the singular values for the Galerkin approximations of the operator $\mathcal{P}_{N}\left(I-R_{a}\right) \mathcal{P}_{N}$. An important issue now is the kind of basis and finite-dimensional projection operators that can be used to obtain manageable matrices for the corresponding approximation operators. The Haar wavelet basis seems to be a good candidate for such a construction, since this basis allows one to obtain a good description for the projection $\mathcal{P}_{N}$. Let $\varphi=\varphi(t), t \in \mathbb{R}$ be the characteristic function of the interval $[0,1)$, and let

$$
h(t):=\left\{\begin{aligned}
1 & \text { if } t \in[0,1 / 2), \\
-1 & \text { if } t \in[1 / 2,1), \\
0 & \text { otherwise. }
\end{aligned}\right.
$$

The Haar wavelet basis of $L_{2}(\mathbb{R})$ comprises the functions

$$
\varphi_{j}:=\varphi(\cdot-j), \quad j \in \mathbb{Z}
$$

and

$$
h_{s, j}:=2^{s / 2} h\left(2^{s / 2} \cdot-j\right), \quad j \in \mathbb{Z}, s \in \mathbb{N}_{0}:=N \cup\{0\}
$$

and each element $f \in L_{2}(\mathbb{R})$ can be represented in the form

$$
f=\sum_{k \in \mathbb{Z}} f_{k} \varphi_{k}+\sum_{s \in \mathbb{N}_{0}} \sum_{k \in \mathbb{Z}} f_{s k} h_{s, k}
$$

with coefficients $f_{k}, f_{s k} \in \mathbb{C}$. Then the system

$$
\Phi:=\left\{\left\{\varphi_{j}, 0 \leq j \leq N-1\right\},\left\{h_{s, j}, s, j \in \mathbb{N}_{0} \quad \text { and } \quad j \leq 2^{s} N-1\right\}\right\}
$$

is an orthonormal basis in the space $L_{2}([0, N])$. Since the support of any element $h_{s, j}$ from the system $\Phi$ is in the interval $\left[j / 2^{s},(j+1) / 2^{s}\right)$, the projection operator $\mathcal{P}_{N}$ admits the following representation:

$$
\mathcal{P}_{N} f=\sum_{k=0}^{N-1} f_{k} \varphi_{k}+\sum_{s=0}^{\infty} \sum_{k=0}^{2^{s} N-1} f_{s k} h_{s, k}, \quad f \in L_{2}(\mathbb{R}) .
$$


Let $P_{n m}^{N}, n, m \in \mathbb{N}_{0}$ be the projections defined by

$$
P_{n m}^{N} f(t)=\left\{\begin{array}{l}
\sum_{k=0}^{n} f_{k} \varphi_{k}+\sum_{s=0}^{m} \sum_{k=0}^{n} f_{s k} h_{s, k}, \quad \text { if } \quad 0 \leq n \leq N-1, \\
\sum_{k=0}^{N-1} f_{k} \varphi_{k}+\sum_{s=0}^{m_{0}-1} \sum_{k=0}^{2^{\left(m_{0}-1\right)} N-1} f_{s k} h_{s, k}+\sum_{s=m_{0}}^{m} \sum_{k=0}^{n} f_{s k} h_{s, k}, \\
\quad \text { if } \quad 2^{m_{0}-1} N \leq n<2^{m_{0}} N, \quad 1 \leq m_{0} \leq m .
\end{array}\right.
$$

It is clear that $\operatorname{im} P_{n m}^{N} \subset \operatorname{im} \mathcal{P}_{N}$ and the sequence $\left(P_{n m}^{N}\right)$ converges strongly to the identity operator on the space $L_{2}([0, N])$ as $n$ and $m$ tend to $\infty$. From now on we choose $n$ in a specific way; that is, for any $m=0,1, \ldots$, let us set $n=2^{m} N-1$ and for simplicity the corresponding projection $P_{n m}^{N}$ is denoted by $P_{m}^{N}$, so

$$
P_{m}^{N} f:=\sum_{k=0}^{N-1} f_{k} \varphi_{k}+\sum_{s=0}^{m} \sum_{k=0}^{2^{m}} f_{s k} h_{s, k} .
$$

To study the solvability of equation (6.1), from $₫ 4$ one may replace the Galerkin approximations of the operator $\left(I-R_{a}^{*}\right)\left(I-R_{a}\right)$ by the Galerkin approximations $P_{m}^{N} \mathcal{P}_{N}\left(I-R_{a}^{*}\right) \mathcal{P}_{N}\left(I-R_{a}\right) \mathcal{P}_{N} P_{m}^{N}$ of the operator $\mathcal{P}_{N}\left(I-R_{a}^{*}\right) \mathcal{P}_{N}\left(I-R_{a}\right) \mathcal{P}_{N}$ of (6.2). Considering the behaviour of the singular values of the corresponding operator matrices, one can get information concerning the kernel dimension of the operator $\mathcal{P}_{N}\left(I-R_{a}\right) \mathcal{P}_{N}$. Before proceeding with this task, let us simplify the operator mentioned.

Lemma 6.1. If $R_{a}: L_{2}(\mathbb{R}) \mapsto L_{2}(\mathbb{R})$ is the refinement operator defined by the right-hand side of (6.1), then $L_{2}([0, N])$ is an invariant subspace for the operator $R_{a}$.

Proof. Actually, this result is not new, but here we give a simple proof using the Haar wavelet basis. Let $T_{l}: L_{2}(\mathbb{R}) \mapsto L_{2}(\mathbb{R}), l \in \mathbb{Z}$ be the operator defined by

$$
T_{l} f(x)=f(2 x-l),
$$

when

$$
R_{a}=\sum_{l=0}^{N} a_{l} T_{l} .
$$

Now it suffices to show that for any basis element $\varphi_{j}$ or $h_{s, j}$ of $L_{2}([0, N])$ and for any operator $T_{l}, l \in 0,1, \ldots, N$, the support of $T_{l} f$ is in $[0, N]$. Starting with the elements $\varphi_{j} \in L_{2}([0, N]), j=0,1, \ldots, N-1$ we notice that

$$
\operatorname{supp}\left\{T_{l} \varphi_{j}\right\}=\left[\frac{l+j}{2}, \frac{l+j+1}{2}\right) \subset\left[0, \frac{N+N-1+1}{2}\right)=[0, N),
$$

and hence $T_{l} \varphi_{j} \in L_{2}([0, N])$. To study the action of the operators $T_{l}$ on the remaining basis elements $h_{s, j} \in L_{2}([0, N])$, we employ equation (4.21), which can be rewritten as

$$
T_{l} h_{s, j}=2^{-1 / 2} h_{s+1,2^{s} l+j} .
$$

If $h_{s, j} \in L_{2}([0, N])$, then

$$
0 \leq j \leq 2^{s} N-1
$$


so that

$$
\begin{aligned}
\operatorname{supp}\left\{T_{l} h_{s, j}\right\}= & \operatorname{supp}\left\{h_{s+1,2^{s} l+j}\right\} \\
& \subset\left[\frac{2^{s} l+j}{2^{s+1}}, \frac{2^{s} l+j+1}{2^{s+1}}\right) \subset\left[0, \frac{2^{s} N+2^{s} N-1+1}{2^{s+1}}\right)=[0, N) .
\end{aligned}
$$

Thus $T_{l}\left(L_{2}([0, N])\right) \subset L_{2}([0, N])$ for any $l=0,1, \ldots, N$, and $L_{2}([0, N])$ is an invariant subspace for the operator $R_{a}$.

Let us now construct the matrices of the operators $\Lambda_{\eta}\left(P_{m}^{N} \mathcal{P}_{N}\left(I-R_{a}^{*}\right) \mathcal{P}_{N}(I-\right.$ $\left.\left.R_{a}\right) \mathcal{P}_{N} P_{m}^{N}\right)$ in the Haar wavelet basis. Since $L_{2}([0, N])$ is an invariant subspace of the operator $R_{a}$, one has

$$
\Lambda_{\eta}\left(P_{m}^{N} \mathcal{P}_{N}\left(I-R_{a}^{*}\right) \mathcal{P}_{N}\left(I-R_{a}\right) \mathcal{P}_{N} P_{m}^{N}\right)=\Lambda_{\eta}\left(P_{m}^{N} \mathcal{P}_{N}\left(I-R_{a}^{*}\right)\left(I-R_{a}\right) \mathcal{P}_{N} P_{m}^{N}\right)
$$

To obtain the matrices $\mathbf{R}_{m}^{N}$ of the operators $\Lambda_{\eta}\left(P_{m}^{N} \mathcal{P}_{N}\left(I-R_{a}^{*}\right)\left(I-R_{a}\right) \mathcal{P}_{N} P_{m}^{N}\right)$, one can proceed as in $\$$. Let us omit unnecessary details and point out only important features of this construction. First of all, note that the matrices $\mathbf{R}_{m}^{N}$ of these operators are again three-diagonal block matrices, although the block structure is different; that is, the dimensions of successive blocks located on the main diagonal are respectively $N, N, 2 N, \ldots, 2^{m} N$. Thus the corresponding identity matrices $I_{n}$ are replaced by the identity matrices $I_{N}, I_{N}, I_{2 N}, \ldots, I_{2^{m}}$. For the other non-zero blocks we have

$$
\begin{gathered}
\mathbf{A}_{-1,-1}^{(1)}=\mathbf{A}_{0,0}^{(1)}=\left(b_{k-j}\right)_{k, j=0}^{N-1}, \\
\mathbf{A}_{s, s}^{(1)}=\left(\sum_{l \in \mathbb{Z}} b_{l} \delta_{2^{s} l+j, k}\right)_{k, j=0}^{2^{s} N-1} \text { if } 1 \leq s \leq m, \\
\mathbf{A}_{-1,-1}^{(2)}=\left(-\frac{1}{2} \sum_{i=j}^{N+j} a_{i-j} p_{i-2 k}\right)_{k, j=0}^{N-1}, \\
\mathbf{A}_{0,-1}^{(2)}=\left(\frac{1}{2} \sum_{i=j}^{N+j}(-1)^{i+1} a_{i-j} p_{2 k-i+1}\right)_{k, j=0}^{N-1}, \\
\mathbf{A}_{s+1, s}^{(2)}=\left(-\sum_{l=0}^{N} 2^{-1 / 2} a_{l} \delta_{2^{s} l+j, k}\right)_{k=0,}^{2^{s+1} N-1, \quad 2^{s} N-1} \quad \text { if } 0 \leq s \leq m-1 .
\end{gathered}
$$

The matrices $\mathbf{A}_{s, r}^{(3)}$ can be obtained by computing the adjoint matrices for $\mathbf{A}_{s, r}^{(2)}$.

The result concerning the solvability of equation (6.1) can now be formulated as follows.

Theorem 6.2. Let $\left(\mathbf{R}_{m}^{N}\right)$ be the sequence of matrices (4.34), where the identity matrices $\mathbf{I}_{n}$ are replaced by the corresponding identity matrices $\mathbf{I}_{2^{s}} \mathrm{~N}$ and the blocks $\mathbf{A}_{s, r}^{(i)}, i=1,2,3$ are described in (6.7)-6.11). Then the refinement operator $I-R_{a}$ : $L_{2}([0, N]) \mapsto L_{2}([0, N])$ is normally solvable and the kernel space of equation (6.1) 


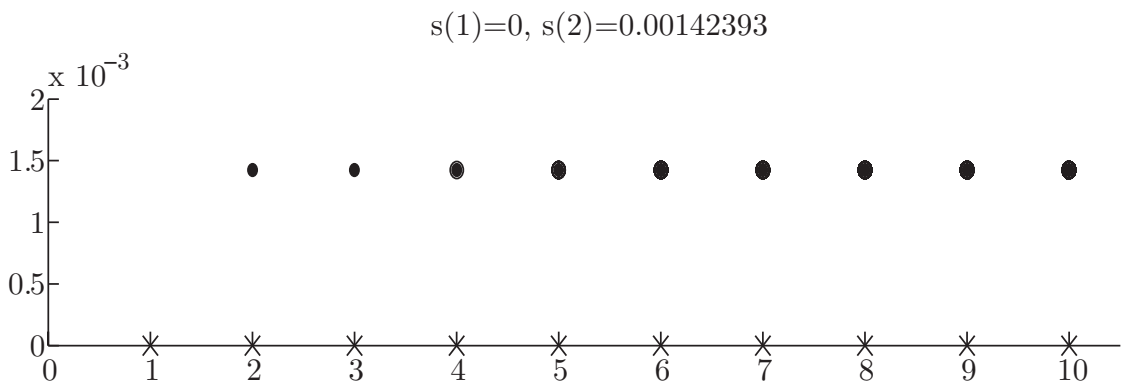

FiguRE 5. Test Equation: HW.

is one-dimensional if and only if the sequences of singular values of matrices $\mathbf{R}_{m}^{N}$ enjoy the 1-splitting property.

To get information about the Fredholmness of the operator $I-R_{a}$, one must consider the sequence of the second smallest singular values.

Theorem 6.3. The refinement operator $I-R_{a}: L_{2}([0, N]) \mapsto L_{2}([0, N])$ of (6.1) is normally solvable if and only if there is an $m_{0} \in \mathbb{N}$ such that the set $\left\{s_{m}(2): m \geq\right.$ $\left.m_{0}\right\}$ is bounded away from zero. In addition, if $I-R_{a}$ is normally solvable, then equation (6.1) has a non-trivial solution if and only if the sequence of the smallest singular values converges to zero.

\section{Numerical tests: COMPaCtly Supported SOlutions}

Let us first consider the Haar refinement equation in the space $L_{2}([0,1])$. The distribution of the smallest singular values is established in Figure 5, where the crosses show the location of the smallest singular values and the points the second smallest singular values.

The integers $m$ on the $X$-axis are related to the projections $P_{m}^{1}$ defined by (6.5). Note that the corresponding matrices $\mathbf{R}_{m}^{1}$ have $2^{m+1}$ rows and $2^{m+1}$ columns. Figure 5 shows that for the Haar refinement equation, the sequence of smallest singular values consists of zeros, but unlike Figure 1 and Figure 2, the sequence of the second smallest singular values is bounded away from zero. Moreover, starting from $m=4$ the singular values $s_{m}(2)$ coincide with the singular values $s_{m}(3)$, and later also with $s_{m}(4)$ and $s_{m}(5)$. Thus the sequences of singular values possess the 1-splitting property. This indicates that the corresponding refinement operator $I-R_{H}$ considered on the space $L_{2}([0,1])$ is normally solvable and, in addition, the graph perfectly reflects the well-known fact that

$$
\operatorname{dim} \operatorname{ker}_{L_{2}([0,1])}\left(I-R_{H}\right)=1 .
$$

A remarkable observation is that, even if the 1-splitting appears to be clearly visible, the lower bound for the sequence of the second smallest singular values is relatively small. As will be seen later, this is the case for most solvable refinement equations, so it can cause additional difficulties in the practical implementation of such a method.

Let us now consider equation (5.1) for the same parameters $k$ as in $\$ 5$. Contrary to the solvability situation in the space $L_{2}(\mathbb{R})$, now this equation is not solvable for any parameter $k \in(1 / \sqrt{2}, \sqrt{2}), k \neq 1$. Thus, if the corresponding operators are 


$$
\mathrm{s}(1)=0.000336584, \mathrm{~s}(2)=0.000336584
$$

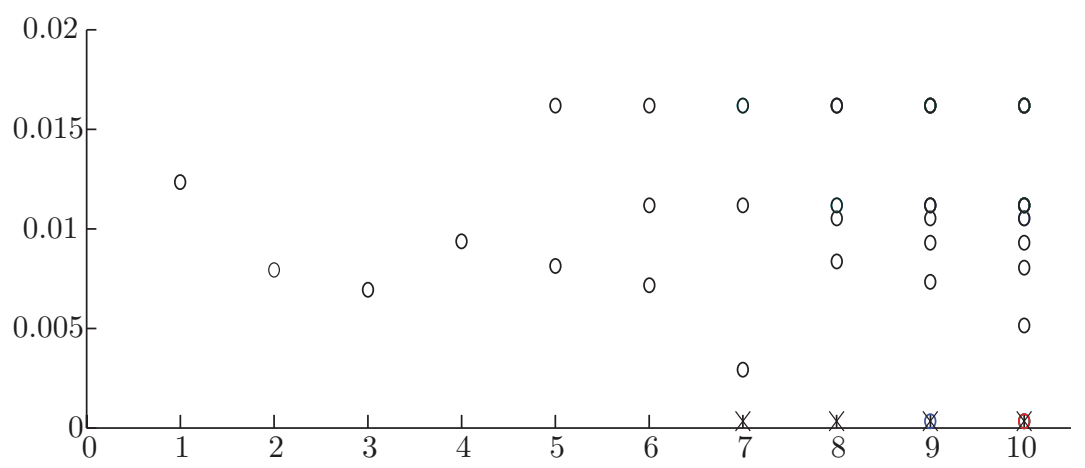

Figure 6. Test Equation: Eqn. (5.1), $k=0.9$.

normally solvable, the sequences of singular values of matrices $\mathbf{R}_{m}^{1}$ should demonstrate the 0 -splitting effect. We start with the case $k=0.9$. In Figure 6 the reader can note that from $2^{8} \times 2^{8}$ matrices to $2^{11} \times 2^{11}$ matrices, the smallest singular values remain on the same distance $d=0.000336584$ from 0 . Let us again emphasize that the stabilization of the sequence of the smallest singular values is achieved at the relatively small number $d=0.000336584$. From $m=9$, the second and some other singular values coincide with the smallest singular value of the corresponding matrices $\mathbf{R}_{m}^{1}$.

Next we turn our attention to equation (5.1) with the parameter $k=0.75$. The distribution of the smallest singular values is presented in Figure 7 . Although the lower bound for the sequence of the smallest singular values is now greater than for $k=0.9$, the 0 -splitting effect in this case is not as conclusive as for the previous example. However, it seems that the stabilization of the sequence $\left(s_{m}(1)\right)$ is already achieved at the value $d=0.00321277$. The reader is recommended to return to this example after considering Figure 11.

Finally, for equation (5.1) we consider the other marginal case: $k=0.5$. As seen in Figure 8 , the sequence of the smallest singular values exhibits a very clear 0 -splitting effect.

$$
\mathrm{s}(1)=0.00321277, \mathrm{~s}(2)=0.00916219
$$

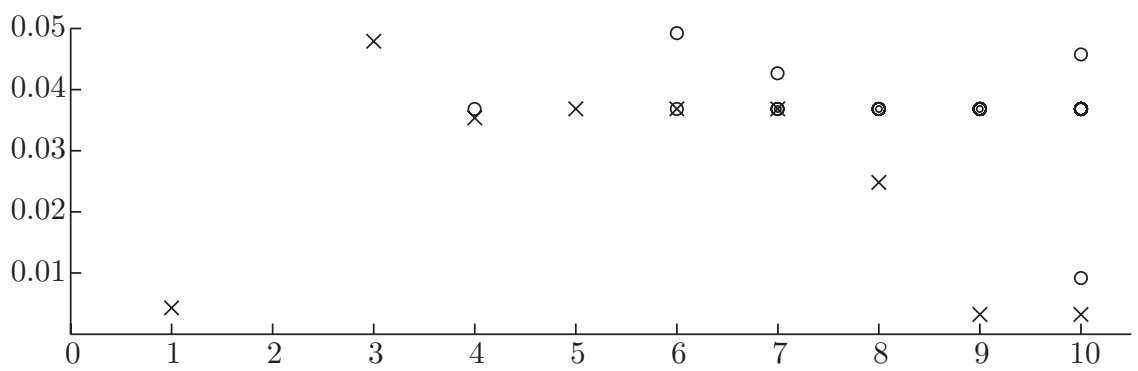

Figure 7. Test Equation: Eqn. (5.1), $k=0.75$. 


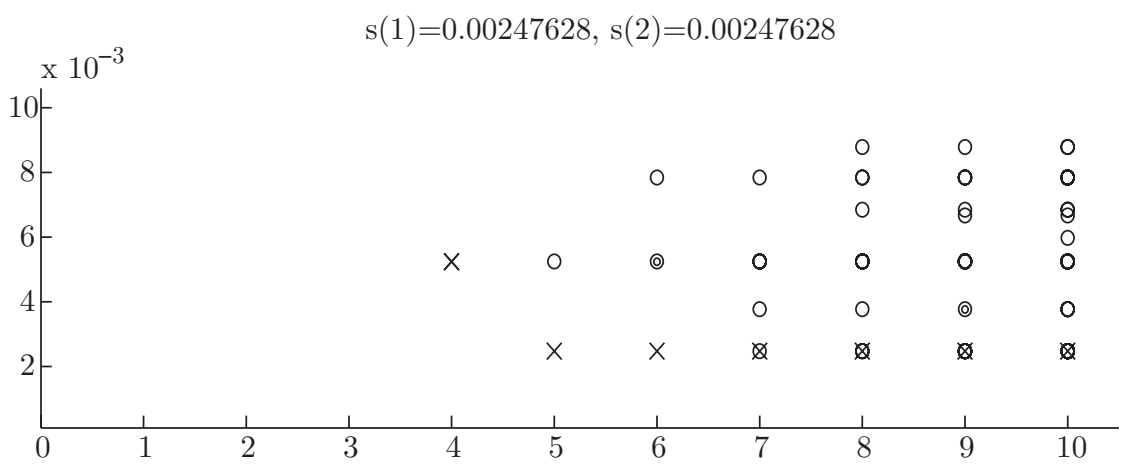

Figure 8. Test Equation: Eqn. (5.1), $k=0.5$.

Thus for refinement equations with two non-zero coefficients, the numerical results properly reflect known results concerning the solvability of such equations in the space $L_{2}([0,1])$.

In the case of refinement equations with a larger number of non-zero coefficients, we point out that they generate the matrices $\mathbf{R}_{m}^{N}, m=1,2, \ldots$ of size $2^{m+1} N \times$ $2^{m+1} N$. Thus in later stages of related computations, obtaining information on singular values becomes more and more challenging. Nevertheless, in some cases there are certain hints about the solvability and Fredholm properties, from the distribution of the smallest singular values. For example, consider the refinement equation

$$
f(x)=\frac{3}{5} f(2 x)+\frac{6}{5} f(2 x-1)+\frac{2}{5} f(2 x-2)-\frac{1}{5} f(2 x-3),
$$

which has a compactly supported continuous solution [9, p. 242]. The distribution of the smallest singular values for the corresponding matrix $\mathbf{R}_{m}^{3}$ is shown in Figure9. (Note that for $m=10$ we show only two smallest singular values.) This graph

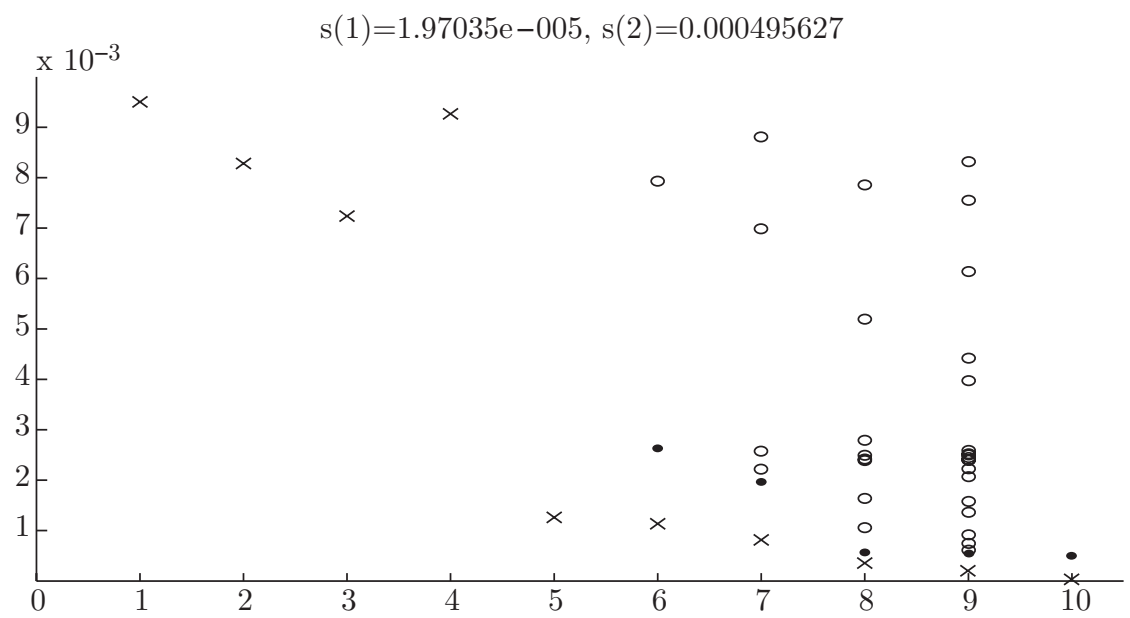

Figure 9. Test Equation: $a_{0}=\frac{3}{5}, a_{1}=\frac{6}{5}, a_{2}=\frac{2}{5}, a_{3}=-\frac{1}{5}$. 
indicates a possible 1-splitting for singular values that corresponds to the fact that equation (7.1) has a non-trivial solution. However, whereas the sequence of the smallest singular values seems to rapidly converge to zero, the sequence of the second smallest singular values has not stabilized. Thus, for $m=8$ the singular value $s_{2^{9}}(2)=0.000567238$, and for $m=10$ the corresponding singular value $s_{2^{11}}(2)$ is 0.000495627 . The difference between these two values is relatively small, and computing singular values for greater values of $m$ could provide better information to decide whether the corresponding refinement operator is normally solvable.

However, there is another possibility for studying the Fredholmness of such operators. Thus if an operator $A \in \mathcal{L}\left(L_{2}([a, b])\right)$ and $B: \mathcal{L}\left(L_{2}([c, d])\right) \mapsto \mathcal{L}\left(L_{2}([a, b])\right)$ is an invertible operator, then one can consider the operator $\widetilde{A}: \mathcal{L}\left(L_{2}([c, d])\right) \mapsto$ $\mathcal{L}\left(L_{2}([c, d])\right)$ defined by

$$
\widetilde{A}=: B^{-1} A B \text {. }
$$

Note that the operators $A$ and $\widetilde{A}$ are normally solvable only simultaneously and

$$
\operatorname{dim} \operatorname{ker}_{L_{2}([c, d])} \widetilde{A}=\operatorname{dim} \operatorname{ker}_{L_{2}([a, b])} A .
$$

Hence, although the corresponding singular values for the operator $\widetilde{A}$ may be different from those for the operator $A$, the sequences of singular values behave similarly. What is important is that such a transformation can increase or decrease the convergence rate of relevant sequences.

To illustrate this idea we set $B=V_{h}$, where

$$
V_{h} f(x)=f(x+h), \quad h \in \mathbb{Z},
$$

is a shift operator. The operator $V_{h}$ is invertible if considered from the space $L_{2}([a+h, b+h])$ to the space $L_{2}([a, b])$. In particular, the equation (6.1) is solvable in the space $L_{2}([0, N])$ if and only if the equation

$$
f(x)=a_{0} f(2 x-h)+a_{1} f(2 x-h-1)+\ldots+a_{N} f(2 x-h-N)
$$

is solvable in the space $L_{2}([h, h+N])$. As was already mentioned, the convergence of the corresponding sequences of singular values is different for different parameters $h$, and this can help in detecting the splitting effect. Let us consider two examples.

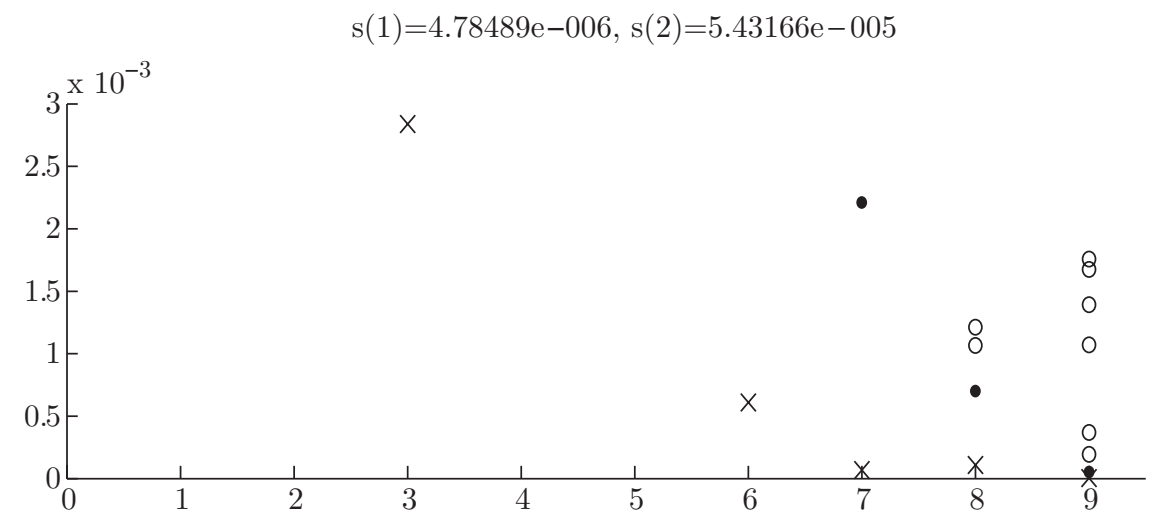

Figure 10. Test Equation: $a_{-2}=\frac{3}{5}, a_{-1}=\frac{6}{5}, a_{0}=\frac{2}{5}, a_{1}=-\frac{1}{5}$. 
The first one is concerned with the equation (7.1). Employing the shift $h=-2$ we obtain the equation

$$
f(x)=\frac{3}{5} f(2 x+2)+\frac{6}{5} f(2 x+1)+\frac{2}{5} f(2 x)-\frac{1}{5} f(2 x-1) .
$$

The behaviour of sequences of the smallest singular values is established in Figure 10. For the sequence of the second smallest singular values one can see rapid convergence to zero. Note that other shifted versions of equation (7.1) also show convergence to zero for the sequence of the second smallest singular values. This can indicate that the corresponding refinement operator may not be normally solvable.

Consider another example where a shifted version of an initial equation turns out to be very helpful. For the equation (5.1) with the parameter $k=0.75$ considered on the space $L_{2}([0,1])$, Figure 7 shows that the stabilization of the sequence of the smallest singular values is achieved on latter stages of computations. On the other hand, choosing $h=-2$ and considering the corresponding shifted versions

$$
f(x)=0.75 f(2 x+2)+0.75 f(2 x+1)
$$

of the equation mentioned we get a much better idea of the kernel of the initial operator. Thus the distribution of the smallest singular values is shown in Figure 11. In contrast to Figure 7 now one can see a strong indication of the 0 -splitting effect.

Thus already a simple shift operator $V_{h}, h \in \mathbb{Z}$ can be used to make the splitting effect more visible. However, other invertible operators can also be adopted for such investigations.

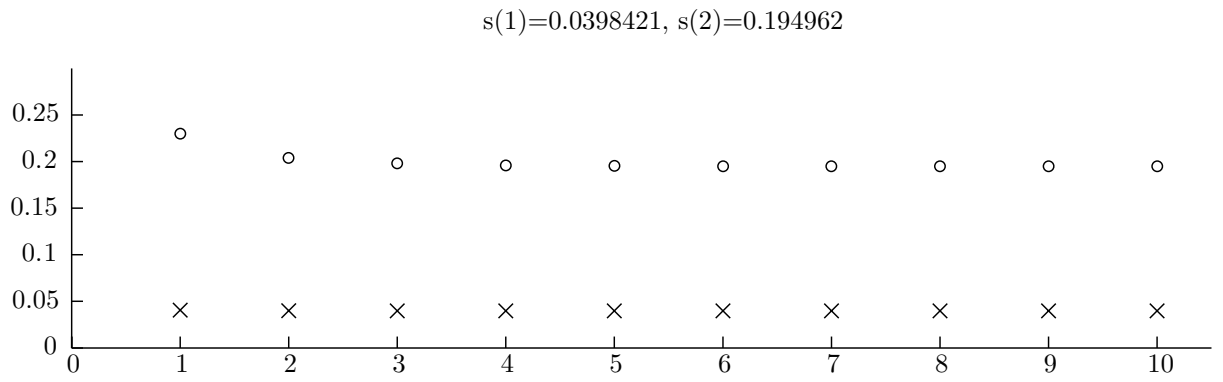

Figure 11. Test Equation: $a_{-2}=0.75, a_{-1}=0.75$.

\section{ACKNowledgements}

The authors wish to thank Vladimir Vasilyev, who set up a MATLAB program, assisted in the numerical experiments, and proposed to use shifted versions of initial equations to make the splitting effect more visible.

\section{REFERENCES}

[1] A.S. Cavaretta, W. Damen, C.A. Micchelli, Stationary subdivisions, Mem. Amer. Math. Soc., 93(1991), 1-186. MR.1079033 (92h:65017)

[2] C.K. Chui, Wavelets: A Mathematical Tool for Signal Processing, SIAM, 1997. MR.1443204 (99b:42012)

[3] A. Cohen, I. Daubechies, A new technique to estimate the regularity of refinable functions, Rev. Mat. Iberoamericana, 12(1996), 527-591. MR1402677 (97g:42025) 
[4] A. Cohen, K. Gröchenig, L.F. Villemoes, Regularity of multivariate refinable functions, Const. Approx., 15(1999), 241-255. MR.1668921 (2000b:42028)

[5] C.K. Chui, An Introduction to Wavelets, Academic Press, 1992. MR.1150048 (93f:42055)

[6] D. Colella, C. Heil, Characterization of scaling functions: Continuous solutions, SIAM J. Matrix Anal., 15(1994), 496-518. MR1266600(95f:26004)

[7] D. Colella, C. Heil, Matrix Refinement Equation: Existence and Uniqueness, J. Fourier Anal. Appl., 2(1996), 363-377. MR1395770 (97k:39021)

[8] I. Daubechies, Orthonormal bases of compactly supported wavelets, Comm. Pure Appl. Math., 41(1988), 909-996. MR951745 (90m:42039)

[9] I. Daubechies, Ten Lectures on wavelets, SIAM, 1992. MR 1162107 (93e:42045)

[10] I. Daubechies, J. Lagarias, Two-scale difference equations, I. Existence and global regularity of solutions, SIAM J. Math. Anal., 22(1991), 388-1410. MR1112515 (92d:39001)

[11] I. Daubechies, J. Lagarias, Two-scale difference equations, II. Local regularity, infinite products of matrices and fractals, SIAM J. Math. Anal., 23(1992), 1031-1079. MR1166574 (93g:39001)

[12] W. Dahmen, C.A. Micchelli, Subdivision algorithms for the generation of box spline surfaces, Comput. Aided Geom. Des., 1(1984), 115-129.

[13] V.D. Didenko, A.A. Korenovsky, S.L. Lee, On the spectral radius of convolution dilation operators, Z. Anal. Anwendungen, 21(2002), 879-890. MR1957302(2004a:39049)

[14] T.B. Dinsenbacher, D.P. Hardin, Nonhomogeneous refinement equations, A. Aldroubi, E.-B. Lin (eds), Wavelets, multiwavelets, and their applications, San Diego, CA, 1997, Contemp. Math. Series, 1998, pp. 117-127. MR.1614717(99a:39055)

[15] I. Gohberg, N. Feldman, Convolution equations and projection methods for their solutions, Akademie-Verlag, Berlin, 1974.

[16] I. Gohberg, N.Ya. Krupnik, Introduction to the theory of one-dimensional singular integral operators, Birkhäuser, 1992. MR545507 (81d:45010)

[17] I. Gohberg, M. Krein, Introduction to the theory of linear nonselfadjoint operators, Nauka, Moscow, 1965. English translation in Translations of Mathematical Monographs, Vol. 18, Amer. Math. Soc., 1969. MR0246142 (39:7447)

[18] R. Hagen, S. Roch, B. Silbermann, $C^{*}$-Algebras and Numerical Analysis, Marcel Dekker. Inc., New York, 2001. MR1792428 (2002g:46133)

[19] B. Han, Solutions in Sobolev spaces of vector refinement equations with general dilation matrix, Adv. Comp. Math., 24(2006), 375-403. MR2222276(2007a:42073)

[20] R.Q. Jia, K.S. Lau, D.X. Zhou, $L_{p}$-solutions of refinement eqiuations, J. Fourier Anal. Appl., 7(2001), 143-167. MR1817673 (2002i:42049)

[21] K.S. Lau, J.R. Wang, Characterization of $L^{p}$ solutions for the two-scale dilation equations, SIAM J. Math. Anal., 26(1995), 1018-1046. MR.1338372 (96f:39004)

[22] S. Mallat, Multiresolution approximation and wavelet orthonormal bases of $L_{2}$, Trans. Amer. Math. Soc., 315(1989), 69-88. MR.1008470 (90e:42046)

[23] D. Malone, Solutions to Dilation Equations, Ph.D. Thesis, University of Dublin, 2000.

$[24]$ D. Malone, $L^{2}(\mathbb{R})$ solutions of dilation equations and Fourier-like transforms, J. Fourier Anal. Appl., 8(2002), 309-317. MR1906255(2003d:42012)

[25] C.A. Micchelli, H. Prautzsch, Uniform refinement of curves, Linear Algebra Appl., 114/115(1989), 841-870. MR986909 (90k:65088)

[26] B. Silbermann, Modified finite sections for Toeplitz operators and their singular values, SIAM J. Matrix. Anal. Appl., 24(2003), 678-692. MR1972674 (2004f:47037)

[27] B. Silbermann, Fredholm theory and numerical linear algebra, Operator Theory Advances and Applications, 160(2005), 403-411. MR2191100 (2006h:47016) 
[28] G. Strang, D.-X. Zhou, Inhomogeneous refinement equations, J. Fourier Anal. Appl., 4(1998), 733-744. MR1666013 (99m:42056)

[29] L.F. Villemoes, Energy moments in time and frequency for two-scale difference equation solutions and wavelets, SIAM J. Math. Anal., 23(1992), 1519-1543. MR1185640 (94c:39002)

Department of Mathematics, Universiti Brunei Darussalam, Bandar Seri Begawan, BE1410, BRUNEI

E-mail address: diviol@gmail.com

Faculty of Mathematics, University of Technology Chemnitz, 09107 Chemnitz, GerMANY

E-mail address: Bernd.Silbermann@mathematik.tu-chemnitz.de 